\title{
Nature and Measure of Entanglement in Quantum Phase Transitions
}

\author{
Rolando Somma, ${ }^{1,2, *}$ Gerardo Ortiz, ${ }^{1}$ Howard Barnum, ${ }^{1}$ Emanuel Knill, ${ }^{1,}+\oplus$ and Lorenza Viola ${ }^{1,+}$ \\ ${ }^{1}$ Los Alamos National Laboratory, Los Alamos, NM 87545, USA \\ ${ }^{2}$ Centro Atómico Bariloche and Instituto Balseiro, \\ 8400 San Carlos de Bariloche, Argentina
}

(Dated: November 13, 2018)

\begin{abstract}
Characterizing and quantifying quantum correlations in states of many-particle systems is at the core of a full understanding of phase transitions in matter. In this work, we continue our investigation of the notion of generalized entanglement [Barnum et al., Phys. Rev. A 68, 032308 (2003)] by focusing on a simple Lie-algebraic measure of purity of a quantum state relative to an observable set. For the algebra of local observables on multi-qubit systems, the resulting local purity measure is equivalent to a recently introduced global entanglement measure [Meyer and Wallach, J. Math. Phys. 43, 4273 (2002)]. In the condensed-matter setting, the notion of Lie-algebraic purity is exploited to identify and characterize the quantum phase transitions present in two exactly solvable models: the Lipkin-Meshkov-Glick model, and the spin-1/2 anisotropic XY model in a transverse magnetic field. For the latter, we argue that a natural fermionic observable-set arising after the Jordan-Wigner transformation, better characterizes the transition than alternative measures based on qubits. This illustrates the usefulness of going beyond the standard subsystem-based framework while providing a global disorder parameter for this model. Our results show how generalized entanglement leads to useful tools for distinguishing between the ordered and disordered phases in the case of broken symmetry quantum phase transitions. Additional implications and possible extensions of concepts to other systems of interest in condensed matter physics are also discussed.

PACS numbers: 3.67.Mn, 03.65.Ud, 05.70.Jk, 05.30.-d,
\end{abstract}

\footnotetext{
*Corresponding author. Email: somma@lanl.gov

${ }^{\dagger}$ Current address: National Institute of Standards and Technology, Boulder, CO 80305, USA

${ }^{\ddagger}$ Current address: Department of Physics and Astronomy, Dartmouth College, Hanover, NH 03755, USA
} 


\section{INTRODUCTION}

Quantum Phase Transitions (QPTs) are qualitative changes occuring in the properties of the ground state of a many-body system due to modifications either in the interactions among its constituents or in their interactions with an external probe [1], while the system remains at zero temperature. Typically, such changes are induced as a parameter $g$ in the system Hamiltonian $H(g)$ is varied across a point at which the transition is made from one quantum phase to a different one. Often some correlation length diverges at this point, in which case the latter is called a quantum critical point. Because thermal fluctuations are inhibited, QPTs are purely driven by quantum fluctuations: fluctuations or correlations in the value of some observable or observables that occur in a pure state. Thus, these are purely quantum phenomena: a classical system in a pure state cannot exhibit correlations. Prominent examples of QPTs are the quantum paramagnet to ferromagnet transition occurring in Ising spin systems under an external transverse magnetic field [2, 3, 4], the superconductor to insulator transition in high- $T_{c}$ superconducting systems, and the superfluid to Mott insulator transition originally predicted for liquid helium and recently observed in ultracold atomic gases [5].

Since entanglement is a property inherent to quantum states and intimately related to quantum correlations [6], one would expect that, in some appropriately defined sense, the entanglement present in the ground state undergoes a substantial change across a point where a QPT occurs. Recently, several authors attempted to better understand QPTs by studying the behavior of different measures of entanglement in the ground state of exactly solvable models (see [7, 18, 9, 10, 11, 12] for representative contributions). Such investigations primarily focused on characterizing entan-

glement using information-theoretic concepts, such as the entropy of entanglement [13] or the concurrence [14], developed for bipartite systems. In particular, a detailed analysis of the twospin concurrence has been carried out for the XY model in a transverse magnetic field [7, 8], whereas the entanglement between a block of nearby spins and the rest of the chain has been considered in [10]. While a variety of suggestive results emerge from such studies, in general a full characterization of the quantum correlations near and at a quantum critical point will not be possible solely in terms of bipartite entanglement. Identifying the entanglement measure or measures that best capture the relevant properties close to criticality, including the critical exponents and universality class of the transition, remains open problems in quantum information and condensed matter theory. 
In Refs. [11, 15], we introduced Generalized Entanglement (GE) as a notion extending the essential properties of entanglement beyond the conventional subsystem-based framework. This notion is general in the sense that it is definable relative to any distinguished subset of observables, without explicit reference to subsystems, which makes it directly applicable to any algebraic language used to describe the system (fermions, bosons, spins, etc.) [16, 17, 18]. Founding the notion on a distinguished set of observables makes it especially well suited to studying QPTs, as our definition makes the existence of GE equivalent to the existence of nonzero correlations or fluctuations in those observables. The basic idea is that any quantum state gives rise to a reduced state on the distinguished subset of observables [19]. These reduced states form a convex set; as with standard quantum states, there are pure (extremal) and mixed (non-extremal) ones [20]. We define a generalized entangled pure state, relative to a subspace of observables, to be one whose reduced state on that subspace is mixed. Although we will have little occasion in the present context to apply it to states that are mixed relative to the full set of observables, we extend this notion to include mixed states by defining a generalized entangled mixed state to be one that cannot be written as a convex combination of generalized-unentangled pure states.

The special case in which the observable set is a Lie algebra is often important in Physics. In a broad class of such algebras described below, the algebra is not only a subspace of operators, but is such that we can define a natural Hermitian projection onto that subspace. Then a simple (global) measure of GE for quantum states is provided by what we call the purity relative to the algebra. This is defined as the squared length of the projection of the Hermitian operator (density matrix) representing the state onto the algebra. As argued in [11], if the correct algebra is chosen, the purity contains information about the relevant quantum correlations that uniquely identify and characterize QPTs of the system.

In this paper, we deepen and expand the analysis initiated in [11], by focusing on the detection of QPTs due to a broken symmetry as revealed by the behavior of an appropriate relative purity of the ground state. In Section $\amalg$ we recall the relevant mathematical setting and the definition of the relative purity as a function of the expectation values of the distinguished observables. In Section III we discuss several examples where the relative purity is seen to provide a natural measure of entanglement. In Section IV we illustrate some physical criteria that are relevant in choosing the appropriate observable subalgebra and using GE as an indicator of QPTs. In Sections $\nabla$ and VI we explicitly characterize the QPTs present in the so-called Lipkin-Meshkov-Glick (LMG) model [21, 22] and in the one-dimensional spin-1/2 anisotropic XY model in a transverse magnetic field, 
respectively. This is done by studying the properties of the purity relative to different algebras of observables in the ground state of both models. We find the relevant critical exponents for these models, and in the case of the anisotropic XY model in a transverse magnetic field, obtain a new "global" disorder parameter, the variance of the number of spinless fermionic excitations in a Jordan-Wigner-transformed representation of the system. Finally, we provide in separate Appendices the details underlying various statements made in the main body of the paper. These include the relationship between standard separability and GE (Appendix A), the GE properties of two special classes of spin states, the cluster and valence bond solid states (Appendix B), the proof of the relationship between the local purity and the Meyer-Wallach entanglement measure (Appendix C), and the semiclassical properties of the LMG model in the thermodynamic limit (Appendix D).

\section{GENERALIZED ENTANGLEMENT AND RELATIVE PURITY}

In the GE approach, entanglement is considered as an observer-dependent property of a quantum state, which is determined by the physically relevant point of view through the expectation values of a distinguished subset of observables. Whenever a preferred decomposition into subsystems is specified in terms of an appropriate (physical or encoded [23, 24, 25, 26]) tensor product structure, GE becomes identical to standard entanglement provided that distinguished observables corresponding to all local actions on the individual subsystems are chosen: in particular, for $\mathcal{H}=\otimes_{i} \mathcal{H}_{i}$ with $\operatorname{dim}\left(\mathcal{H}_{i}\right)=d_{i}$, standard entanglement of states in $\mathcal{H}$ is recovered as GE relative to $\mathfrak{h}_{l o c}=\oplus_{i} \mathfrak{s u}\left(d_{i}\right)$ [11, 15] (see also Appendix A]. In fact, the subsystems relative to which standard entanglement is defined (whether directly identifiable with physical degrees of freedom or related to "encoded" or "virtual" ones) are always understandable in terms of appropriate (associative) algebras of local observables. This has been observed before, e.g. in [25, 26] (see also [27] for a recent analysis). However, it is important to realize that the GE notion genuinely extends the standard entanglement definition, and does not coincide with or reduce to it in general. On one hand, this may be appreciated by noticing that even for situations where a subsystem partition is naturally present, states which are manifestly separable relative to such a partition may possess GE relative to an algebra different from $\mathfrak{h}_{l o c}$ (see the two spin-1 example of Section III). On the other hand, as also emphasized in [11], GE is operationally meaningful in situations where no physically accessible decomposition into subsystems exists, thus making conventional entanglement 
not directly definable.

\section{A. Relative purity for faithfully represented Lie algebras}

As mentioned in the Introduction, we will focus on the case where the distinguished observables form a Lie algebra $\mathfrak{h}$ of linear operators, acting on a finite-dimensional state space $\mathcal{H}$ for the system of interest, $\mathcal{S}$. (Note that we will not usually distinguish between the abstract Lie algebra isomorphic to $\mathfrak{h}$, and the concrete Lie algebra $\mathfrak{h}$ of operators that faithfully represents it on $\mathcal{H}$.) We will assume $\mathfrak{h}$ to be a real Lie algebra consisting of Hermitian operators, with the bracket of two linear operators $X$ and $Y$ being given by

$$
[X, Y]=i(X Y-Y X)
$$

In this way, operators in $\mathfrak{h}$ can be directly associated with physical observables. For the same reason, we will also use a slightly nonstandard (but familiar to physicists) notion of the Lie group generated by $\mathfrak{h}$, involving the map $X \mapsto e^{i X}$ instead of the mathematicians' $X \mapsto e^{X}$, for $X \in$ $\mathfrak{h}$. No assumption that the Lie algebra acts irreducibly on $\mathcal{H}$ (i.e., that it admits no nontrivial invariant subspaces) will be made, but important consequences of making such an assumption will be discussed. We will also assume the Lie algebra to be closed under Hermitian conjugation. This implies that it is a reductive algebra (not to be confused with reducibility of the representation). In our context, a reductive Lie algebra is best thought of as the product (direct sum, as a vector space) of a finite number of simple Lie algebras, and a finite number of copies of a one-dimensional Abelian Lie algebra. A simple Lie algebra is a non-Abelian one possessing no nontrivial ideals, where an ideal is a subalgebra invariant under commutation with anything in the algebra; the relevant property of ideals here is that they can be quotiented out of the algebra, allowing it to be written as a nontrivial product of ideal and quotient; thus simple Lie algebras are non-Abelian ones that cannot be decomposed into factors, so the factorization used in defining reductive Lie algebras above is maximal. The product (direct vector-space sum) of a finite number of simple Lie algebras is called semisimple, and thus a reductive algebra is the product of a semisimple and an Abelian part. The reader is referred to [28, 29, 30, 31] for relevant background on Lie algebras and their representation theory. As this subsection unfolds, we will summarize much of this representation theory in a way suited to our needs, and the reader should concentrate on understanding the content of the statements, and not vex him or herself unduly about understanding why they are true. 
We will consider pure quantum states of $\mathcal{S},|\psi\rangle \in \mathcal{H}$, as well as mixed quantum states of $\mathcal{S}$, described by density matrices $\rho$ acting on $\mathcal{H}$. Since $\mathfrak{h}$ was assumed closed under Hermitian conjugation, the projection of a quantum state $\rho$ onto $\mathfrak{h}$ with respect to the trace inner product is uniquely defined. Let $\mathcal{P}_{\mathfrak{h}}$ denote the projection map, $\rho \mapsto \mathcal{P}_{\mathfrak{h}}(\rho)$. If $\rho$ is a pure state, $\rho=$ $|\psi\rangle\langle\psi|$, the purity of $|\psi\rangle$ relative to $\mathfrak{h}$ (or $\mathfrak{h}$-purity) is defined as the squared length of the projection according to the trace inner product norm $\lfloor 15]$; that is

$$
P_{\mathfrak{h}}(|\psi\rangle)=\operatorname{Tr}\left[\left(\mathcal{P}_{\mathfrak{h}}(|\psi\rangle\langle\psi|)\right)^{2}\right]
$$

The $\mathfrak{h}$-purity may be explicitly evaluated upon selecting an operator basis $\mathcal{B}=\left\{A_{1}, \ldots, A_{L}\right\}$ for h. By assuming the $A_{\alpha}$ to be Hermitian,

$$
A_{\alpha}=A_{\alpha}^{\dagger}
$$

and orthogonal,

$$
\operatorname{Tr}\left(A_{\alpha} A_{\beta}\right)=\delta_{\alpha, \beta}
$$

Eq. (2) may be rewritten as

$$
P_{\mathfrak{h}}(|\psi\rangle)=\operatorname{Tr}\left[\sum_{\alpha, \beta=1}^{L} \operatorname{Tr}\left(A_{\alpha} \rho\right) \operatorname{Tr}\left(A_{\beta} \rho\right) A_{\alpha} A_{\beta}\right]=\sum_{\alpha=1}^{L}\left\langle A_{\alpha}\right\rangle^{2},
$$

where $\left\langle A_{\alpha}\right\rangle$ denotes the expectation value of the observable $A_{\alpha}$ in the pure state $|\psi\rangle$.

An important property following is that the $\mathfrak{h}$-purity is invariant under group transformations: if a new basis for $\mathfrak{h}$ is introduced by letting $\tilde{A}_{\alpha}=D^{\dagger} A_{\alpha} D$, with $D=\exp \left(i \sum_{\beta=1}^{L} t_{\beta} A_{\beta}\right), D^{\dagger} D=\mathbb{1}$, and $t_{\beta}$ real numbers, then one finds

$$
\tilde{P}_{\mathfrak{h}}(|\psi\rangle)=\sum_{\alpha=1}^{L}\left\langle\tilde{A}_{\alpha}\right\rangle^{2}=\sum_{\alpha=1}^{L}\left\langle A_{\alpha}\right\rangle^{2}=P_{\mathfrak{h}}(|\psi\rangle) .
$$

Sometimes it is useful to introduce a common normalization factor $\mathrm{K}$ in order to set the maximum value of the purity to 1 , in which case Eq. (5) becomes

$$
P_{\mathfrak{h}}(|\psi\rangle)=\mathrm{K} \sum_{\alpha=1}^{L}\left\langle A_{\alpha}\right\rangle^{2}
$$

As mentioned earlier, a pure quantum state $|\psi\rangle$ is defined to be generalized entangled (generalized unentangled) relative to $\mathfrak{h}$ if it induces a mixed (pure) state on that set of observables. When $\mathfrak{h}$ is a complex semisimple Lie algebra acting irreducibly on $\mathcal{H}$, it was shown in [15] (Theorem 
14, part (4)) that $|\psi\rangle$ is generalized unentangled with respect to $\mathfrak{h}$ if and only if it has maximum $\mathfrak{h}$-purity, and generalized entangled otherwise. Under the same assumptions, the abovementioned Theorem (part (3)) also leads to the identification of the generalized unentangled pure states as the generalized coherent states (GCSs) associated with $\mathfrak{h}[32,33,34]$. In other words, all generalized unentangled states are in the (unique) orbit of a minimum weight state of $\mathfrak{h}$ (taken as a reference state) under the action of the Lie group. Remarkably, GCSs are known to possess minimum invariant uncertainty, $(\Delta F)^{2}(|\psi\rangle)=\sum_{\alpha}\left[\left\langle A_{\alpha}^{2}\right\rangle-\left\langle A_{\alpha}\right\rangle^{2}\right][35$, 36], so that, similar to the familiar harmonic-oscillator ones, they may be regarded in some sense as closest to "classical" states.

Our characterization theorem for generalized unentangled states on irreducible representations used some standard facts from the theory of semisimple Lie algebras and their representations that will also be useful in the discussion of reducible representations in the next subsection. These are the existence of Cartan (in the semisimple context, maximal Abelian) subalgebras, their conjugacy under the action of the Lie group associated with the algebra, and the fact that any finitedimensional representation, given a choice of Cartan subalgebra (CSA), decomposes into mutually orthogonal "weight spaces," which are simultaneously eigenspaces of all CSA elements. The map from CSA elements to their eigenvalues on a given weight space is a linear functional on the CSA called the "weight" of that weight space. The theorem also uses the observation that the projection of the state into the Lie algebra is necessarily a Hermitian element of that algebra, hence semisimple (diagonalizable), hence belonging to some CSA, which we call its supporting CSA. Frequently, semisimple Lie algebras are presented by giving a Cartan-Weyl basis, consisting of a set of commuting, jointly diagonalizable operators that generate a CSA of the algbera, and a set of so-called "Weyl operators" that are non-diagonalizable, and act to take a state in one weight space to a state in another (or else annihilate it): in physical examples these are often called "raising and lowering operators." Normalized states correspond to normalized linear functionals on the Lie algebra; when a Cartan-Weyl basis for the algebra is chosen such that the CSA distinguished by the basis is the supporting CSA for a given state, the state is zero except on the CSA part of the basis. On the CSA, the state is some convex combination of the weights, that is an element of the weight polytope (which is defined as the convex hull of the weights). So it turns out that extremal states on the Lie algebra correspond to extremal points of the weight polytope. This applies regardless of whether the representation is irreducible or not. For irreducible representations (irreps), the extremal points of the weight polytope are also highest-weight states of the irrep. Reducible representations are discussed in the next subsection (along with some comments 
on reductive algebras).

In preparation for that, we introduce another aspect of standard Lie theory: the Weyl group. Besides being able to take any CSA to any other CSA, the Lie group also acts on the weight polytope for a given CSA, by reflections in a set of hyperplanes through the origin. The group these generate is called the Weyl group. Considered together, the hyperplanes divide the weight space into a set of convex cones, sometimes called Weyl chambers, whose points are at the origin, and whose union with the hyperplanes is the entire space. Any such cone can be mapped to any other via the Weyl group action, and the weight polytope of the representation is the convex hull of the Weyl group orbits of the weights in the closure of any single Weyl chamber.

\section{B. Irreducibly vs reducibly represented Lie algebras}

It is important to realize that the relationships just mentioned between maximal purity, generalized coherence, and generalized unentanglement established for a pure state relative to an irreducibly represented algebra $\mathfrak{h}$ do not automatically extend to the case where $\mathfrak{h}$ acts reducibly on $\mathcal{H}$. We will discuss semisimple algebras first and then, because the algebra we use to analyze the LMG model is Abelian, the case of reductive algebras.

If $\mathfrak{h}$ is semisimple, a generic finite-dimensional representation of $\mathfrak{h}$ may be decomposed as a direct sum of irreducible invariant subspaces, $\mathcal{H} \simeq \oplus_{\ell} \mathcal{H}_{\ell}$, with each of the $\mathcal{H}_{\ell}$ being in turn the direct sum of its weight spaces. Every irrep appearing in the decomposition has a highest (or lowest) weight, and for each of these irreps, there is a manifold of GCSs for the irrep constructed as the orbit of a highest weight state for that irrep. The weight polytope for the reducible representation will be the convex hull of those for all the irreps contained in it. Because of this, the GCSs for these irreps will not, in general, all satisfy the extremality property that defines generalized unentangled states. This reflects the fact that even for a state belonging to a specific $\mathfrak{h}$-irrep, GE is a property which depends in general on how the state relates to the whole representation, not solely the irrep. Nor is there necessarily a single weight, for one of the constituent irreps, that generates (as the convex hull of the Weyl group orbit) the weight polytope of the reducible representation. Indeed, the extremal weights in the weight polytope, which correspond to generalized unentangled states, need not all have the same length. Since this squared length is the $\mathfrak{h}$-purity (as defined in Eq. (5))

of the corresponding state, it is thus no longer the case that all generalized unentangled states have maximal Lie-algebraic purity. However, maximal purity remains a sufficient, though no longer a 
necessary, condition for generalized unentanglement. If the algebra is reductive, the expectations of a maximal commutative subalgebra now include ones for the Abelian part of the algebra, i.e. operators that commute with the entire algebra. These must be proportional to the identity on each irrep, but may have different eigenvalues (possibly degenerate) on different irreps. States on this algebra then involve not just weights for the semisimple part of the algebra, but expectation values for the Abelian part of the algebra as well. These can distinguish different subsets of the irreps, and so irreps whose highest weight for the semisimple part is not extremal for the semisimple part, may become extremal (generalized unentangled) in the full reductive algebra. However, maximal quadratic purity will remain a sufficient, though in general still not necessary, condition for a state being generalized unentangled.

More intuition about GE, purity, and GCSs may be gained from simple examples. Consider a physical system which is composed of two spin-1/2s (namely, two qubits), and let them be labeled by $A, B$, with $\mathcal{H}=\mathcal{H}_{A} \otimes \mathcal{H}_{B}=\mathbb{C}^{4}$, and corresponding $\mathfrak{s u}(2)$ generators $\sigma_{\alpha}^{A}, \sigma_{\alpha}^{B}, \alpha \in\{x, y, z\}$. Consider GE relative to a global representation of $\mathfrak{s u}(2)$, whose total-spin generators are $J_{\alpha}=$ $\sigma_{\alpha}^{A}+\sigma_{\alpha}^{B}$. This representation splits into two irreps, the one-dimensional singlet representation with $J=0$ and the three-dimensional triplet representation with $J=1$. The generalized unentangled states relative to this representation of $\mathfrak{s u}(2)$ are those for which there exists an $\alpha$ such that the state is a \pm 1 eigenstate of $J_{\alpha}$. With respect to the CSA $\mathfrak{c}=\left\{J_{z}\right\}$, those are the states $|\uparrow, \uparrow\rangle,|\downarrow, \downarrow\rangle$, which are also GCSs (with purity equal to 1). No generalized unentangled state is contained in the singlet irrep. In particular, neither the spin-zero state in the triplet, nor that which spans the singlet, are generalized unentangled (they both have purity equal to 0 ), nor are they on highest-weight orbits (thus GCSs).

As another example consider a single spin-1 system, whose state space $\mathcal{H}=\mathbb{C}^{3}$ carries an irrep of $\mathfrak{s u}(2)[11]$. In this case, for any choice of spin direction (say $z$ ) only the $J_{z}= \pm 1$ eigenstates are generalized coherent. There is also a one-dimensional $J_{z}=0$ eigenspace. The maximal-purity states are also the highest-weight states; however, the pure $J_{z}=0$ eigenstate is not a GCS, has zero purity, and is generalized entangled. If, for the same system, a distinguished algebra $\mathfrak{s o}(2)$ generated by $J_{z}$ alone is chosen, then the representation reduces as the direct sum of the three invariant one-dimensional subspaces corresponding to $J_{z}=1,0,-1$. In this case, three different orbits exist in the representation, each of them consisting of only one state up to phases. However, only the states with $\left|J_{z}\right|=1$ are extremal, whereas the state with $J_{z}=0$ is not: as one can easily verify from the fact that the reduced state is now just the expectation value of $J_{z}$, an equal mixture 
of a $J_{z}=1$ and a $J_{z}=-1$ state has the same reduced state as a $J_{z}=0$ state, so the latter remains, as in the irreducible case, generalized entangled.

A generalization of the latter example, which is relevant to the LMG model we will study in Section $\nabla$ is the case of a spin- $J$ system with a distinguished Abelian subalgebra generated by $J_{z}$. Again, one can see that only the states with maximal magnitude of $J_{z}$ are generalized unentangled, and only they have maximal purity.

By definition, note that the relative purity and the invariant uncertainty functionals as defined in the previous section relate to each other via

$$
(\Delta F)^{2}=\left\langle\mathcal{C}_{2}\right\rangle-P_{\mathfrak{h}},
$$

where $\mathcal{C}_{2}$ denotes the quadratic Casimir invariant of the Lie algebra and $P_{\mathfrak{h}}$ is given by Eq. (5) (prior to rescaling). Because, by standard representation theory, $\mathcal{C}_{2}=c_{\ell} \mathbb{1}$, with $c_{\ell} \in \mathbb{R}$ within each irrep, relative purity and invariant uncertainty essentially provide the same information if $\mathfrak{h}$ acts irreducibly. This, however, is no longer true in general in the reducible case. In the above two-spin-1/2 example, for instance, the two measures agree on the singlet sector; for triplet states, $J(J+1)=2$, thus the invariant uncertainty value is 1 (same as $P_{\mathfrak{h}}$ ) for $\left|J_{z}\right|=1$ (generalized unentangled) states, whereas it yields 2 for the (zero-purity) state with $J_{z}=0$ in the triplet sector.

\section{Extension to mixed states}

For mixed states on $\mathcal{H}$, the direct generalization of the squared length of the projection onto $\mathfrak{h}$ as in Eq. (2) does not give a GE measure with well-defined monotonicity properties under appropriate generalizations of the LOCC semigroup of transformations [15]. A proper extension of the quadratic purity measure defined in the previous section for pure states to mixed states may be naturally obtained via a standard convex roof construction. If $\rho=\sum_{s} p_{s}\left|\psi_{s}\right\rangle\left\langle\psi_{s}\right|$, with $\sum_{s} p_{s}=1$ and $\sum_{s} p_{s}^{2}<1$, the latter is obtained by calculating the maximum $\mathfrak{h}$-purity (minimum entanglement) over all possible convex decompositions $\left\{p_{s},\left|\psi_{s}\right\rangle\right\}$ of the density operator $\rho$ as a pure-state ensemble. In general, similarly to what happens for most mixed-state entanglement measures, the required extremization makes the resulting quantity very hard to compute.

While a more expanded discussion of mixed-state GE measures is given in [15], we focus here on applying the notion of GE to characterize QPTs in different lattice systems. Because the latter take place in the limit of zero temperature, the ground state of the system may be assumed to be 
pure under ideal conditions. Accordingly, Eq. (7) will suffice for our current purposes.

\section{RELATIVE PURITY AS A MEASURE OF ENTANGLEMENT IN DIFFERENT QUANTUM SYSTEMS}

We now apply the concept of relative purity to different physical systems in order to understand its meaning as a measure of entanglement for pure quantum states. First, we will concentrate on spin systems, showing that for particular subsets of observables, the $\mathfrak{h}$-purity can be reduced to the usual notion of entanglement: the pure quantum states that can be written as a product of states of each party will be generalized unentangled. However, for other physically natural choices of observable sets, this is no longer the case. Next, we study the h-purity as a measure of entanglement for fermionic systems, since this is a good starting point for the analysis of the QPT present in the anisotropic XY model in a transverse magnetic field (Section VI). In particular, we show that if a fermionic state can be represented as a single Slater determinant, it is generalized unentangled relative to the Lie algebra $\mathfrak{u}(N)$, which is built from bilinear products of fermionic operators. These examples illustrate how the concept and measure of GE is applicable to systems described by different operator languages, in preparation for the study of QPTs.

Let us introduce the following representative quantum states for $N$ spins of magnitude $S$ :

$$
\begin{aligned}
\left|\mathrm{F}_{S}^{N}\right\rangle & =|S, S, \cdots, S\rangle, \\
\left|\mathrm{W}_{S}^{N}\right\rangle & =\frac{1}{\sqrt{N}} \sum_{i=1}^{N}\left|S, \cdots, S,(S-1)_{i}, S, \cdots, S\right\rangle, \\
\left|\mathrm{GHZ}_{S}^{N}\right\rangle & =\frac{1}{\sqrt{2 S+1}} \sum_{l=0}^{2 S}|S-l, S-l, \cdots, S-l\rangle,
\end{aligned}
$$

where the product state $\left|S_{1}, S_{2}, \cdots, S_{N}\right\rangle=\left|S_{1}\right\rangle_{1} \otimes\left|S_{2}\right\rangle_{2} \otimes \cdots \otimes\left|S_{N}\right\rangle_{N}$, and $\left|S_{i}\right\rangle_{i}$ denotes the state of the $i$ th party with $z$-component of the spin equal to $S_{i}$ (defining the relevant computational basis for the $i$ th subsystem).

\section{A. Two-spin systems}

For simplicity, we begin by studying the GE of a two-qubit system (two spin-1/2s), where the

most general pure quantum state can be written as $|\psi\rangle=a\left|\frac{1}{2}, \frac{1}{2}\right\rangle+b\left|\frac{1}{2},-\frac{1}{2}\right\rangle+c\left|-\frac{1}{2}, \frac{1}{2}\right\rangle+d\left|-\frac{1}{2},-\frac{1}{2}\right\rangle$, with the complex numbers $a, b, c$, and $d$ satisfying $|a|^{2}+|b|^{2}+|c|^{2}+|d|^{2}=1$. The traditional 
measures of pure-state entanglement in this case are well understood, indicating that the Bell states $\left|\mathrm{GHZ}_{\frac{1}{2}}^{2}\right\rangle[37]$ (and its local spin rotations) are maximally entangled with respect to the local Hilbert space decomposition $\mathcal{H}_{1} \otimes \mathcal{H}_{2}$. On the other hand, calculating the purity relative to the (irreducible) Lie algebra of all local observables $\mathfrak{h}=\mathfrak{s u}(2)_{1} \oplus \mathfrak{s u}(2)_{2}=\left\{\sigma_{\alpha}^{i} ; i: 1,2 ; \alpha=x, y, z\right\}$ classifies the pure two-spin-1/2 states in the same way as the traditional measures do (see Fig. 11). Here, the operators $\sigma_{\alpha}^{1}=\sigma_{\alpha} \otimes \mathbb{1}$ and $\sigma_{\alpha}^{2}=\mathbb{1} \otimes \sigma_{\alpha}$ are the Pauli operators acting on spin 1 and 2, respectively, and

$$
\mathbb{1}=\left(\begin{array}{ll}
1 & 0 \\
0 & 1
\end{array}\right), \sigma_{x}=\left(\begin{array}{ll}
0 & 1 \\
1 & 0
\end{array}\right), \sigma_{y}=\left(\begin{array}{cc}
0 & -i \\
i & 0
\end{array}\right), \sigma_{z}=\left(\begin{array}{cc}
1 & 0 \\
0 & -1
\end{array}\right)
$$

in the basis where $|+1 / 2\rangle=|\uparrow\rangle=\left(\begin{array}{l}1 \\ 0\end{array}\right)$ and $|-1 / 2\rangle=|\downarrow\rangle=\left(\begin{array}{l}0 \\ 1\end{array}\right)$. In this case, Eq. (7) simply gives

$$
P_{\mathfrak{h}}(|\psi\rangle)=\frac{1}{2} \sum_{i, \alpha}\left\langle\sigma_{\alpha}^{i}\right\rangle^{2}
$$

where Bell's states are maximally entangled $\left(P_{\mathfrak{h}}=0\right)$ and product states of the form $|\psi\rangle=$ $\left|\phi_{1}\right\rangle_{1} \otimes\left|\phi_{2}\right\rangle_{2}$ (GCSs of the local algebra $\mathfrak{h}$ above) are generalized unentangled, with maximum purity. Therefore, the normalization factor $\mathrm{K}=1 / 2$ may be obtained by setting $P_{\mathfrak{h}}=1$ in such a product state. As explained in Section $\amalg P_{\mathfrak{h}}$ is invariant under group operations, i.e., in this case, local rotations. Since all GCSs of $\mathfrak{h}$ belong to the same orbit generated by the application of group operations to a particular product state (a reference state like $\left|\frac{1}{2}, \frac{1}{2}\right\rangle=|\uparrow, \uparrow\rangle$ ), they all consistently have maximum $\mathfrak{h}$-purity $\left(P_{\mathfrak{h}}=1\right)$.

Another important insight may be gained by calculating the purity relative to the algebra of all observables for the system, $\mathfrak{h}=\mathfrak{s u}(4)=\left\{\sigma_{\alpha}^{i}, \sigma_{\alpha}^{1} \otimes \sigma_{\beta}^{2} ; i=1,2 ; \alpha, \beta=x, y, z\right\}$ in this case. One finds that any two spin-1/2 pure state $|\psi\rangle$ (including Bell's states) is then generalized unentangled $\left(P_{\mathfrak{h}}=1\right.$, see also Fig. 11). This property is a manifestation of the relative nature of GE, as considering the set of all observables as being physically accessible is equivalent to not making any preferred subsystem decomposition. Accordingly, in this case any pure quantum state becomes a GCS of $\mathfrak{s u}(4)$.

In Fig. 1 we also show the GE for systems of two parties of spin- $S$ relative to different algebras. We observe that the purity reduces again to the traditional concept of entanglement for higher spin if it is calculated relative to the (irreducible) Lie algebra of all local observables $\mathfrak{h}=\mathfrak{s u}(2 S+1)_{1} \oplus$ $\mathfrak{s u}(2 S+1)_{2}$. For example, if we are interested in distinguishing product states from entangled states in a two-spin-1s system, we need to calculate the purity relative to the (irreducible) algebra 


\begin{tabular}{|c|c|c|c|}
\hline$\frac{1}{2}$ & $\mathfrak{s u}(2) \oplus \mathfrak{s u}(2)$ & & $\mathfrak{s u}(4)$ \\
\hline & $\begin{array}{l}1-\left|F_{\frac{1}{2}}^{2}\right\rangle \\
\frac{1}{4}----\frac{\left|\frac{1}{2}, \frac{1}{2}\right\rangle+\sqrt{3}\left|-\frac{1}{2},-\frac{1}{2}\right\rangle}{2} \\
0 \\
\left|G H Z_{\frac{1}{2}}^{2}\right\rangle\end{array}$ & & $1 \longrightarrow\left|F_{\frac{1}{2}}^{2}\right\rangle ;\left|G H Z_{\frac{1}{2}}^{2}\right\rangle ;$ \\
\hline 1 & $\mathfrak{s u}(2) \oplus \mathfrak{s u}(2)$ & $\mathfrak{s u}(3) \oplus \mathfrak{s u}(3)$ & $\mathfrak{s u}(9)$ \\
\hline & $\begin{array}{l}1-\left|F_{1}^{2}\right\rangle \\
\frac{1}{4}----\frac{|1,1\rangle+\sqrt{3}|-1,-1\rangle}{2} \\
\left.0-G H Z_{1}^{2}\right\rangle ;|0,0\rangle\end{array}$ & $\begin{array}{l}1 \longrightarrow\left|F_{1}^{2}\right\rangle ;|0,0\rangle \\
\frac{7}{16}----\frac{|1,1\rangle+\sqrt{3}|-1,-1\rangle}{2} \\
0 \longrightarrow\left|\mathrm{GHZ}_{1}^{2}\right\rangle\end{array}$ & $1 \longrightarrow \frac{\left.F_{1}^{2}\right\rangle ;|0,0\rangle ;}{\left|G H Z_{1}^{2}\right\rangle ; \cdots}$ \\
\hline$S$ & $\mathfrak{s u}(2) \oplus \mathfrak{s u}(2)$ & $\mathfrak{s u}(2 S+1) \oplus \mathfrak{s u}(2 S+1)$ & $\mathfrak{s u}\left([2 S+1]^{2}\right)$ \\
\hline & $\left|\mathrm{GHZ}_{S}^{2}\right\rangle$ & $\begin{array}{c}\left.1 \longrightarrow F_{S}^{2}\right\rangle \\
\\
\left|G H Z_{S}^{2}\right\rangle\end{array}$ & $\left.1 \coprod_{S}^{2}\right\rangle ;\left|G H Z_{S}^{2}\right\rangle$ \\
\hline
\end{tabular}

FIG. 1: Purity relative to different possible algebras for a two-spin- $S$ system. The quantum states $\left|G H Z_{S}^{2}\right\rangle$ and $\left|F_{S}^{2}\right\rangle$ are defined in Eqs. (9.

$\mathfrak{h}=\mathfrak{s u}(3)_{1} \oplus \mathfrak{s u}(3)_{2}=\left\{\lambda_{\alpha}^{1} \otimes \mathbb{1}^{2}, \mathbb{1}^{1} \otimes \lambda_{\alpha}^{2}(1 \leq \alpha \leq 8)\right\}$, where the $3 \times 3$ Hermitian and traceless 
matrices $\lambda_{i}$ are the well known Gell-Mann matrices [28]:

$$
\begin{aligned}
& \lambda_{1}=\frac{1}{\sqrt{2}}\left(\begin{array}{lll}
0 & 1 & 0 \\
1 & 0 & 0 \\
0 & 0 & 0
\end{array}\right) ; \lambda_{2}=\frac{1}{\sqrt{2}}\left(\begin{array}{ccc}
0 & -i & 0 \\
i & 0 & 0 \\
0 & 0 & 0
\end{array}\right) \\
& \lambda_{3}=\frac{1}{\sqrt{2}}\left(\begin{array}{ccc}
1 & 0 & 0 \\
0 & -1 & 0 \\
0 & 0 & 0
\end{array}\right) ; \lambda_{4}=\frac{1}{\sqrt{2}}\left(\begin{array}{lll}
0 & 0 & 1 \\
0 & 0 & 0 \\
1 & 0 & 0
\end{array}\right) \\
& \lambda_{5}=\frac{1}{\sqrt{2}}\left(\begin{array}{ccc}
0 & 0 & -i \\
0 & 0 & 0 \\
i & 0 & 0
\end{array}\right) ; \lambda_{6}=\frac{1}{\sqrt{2}}\left(\begin{array}{ccc}
0 & 0 & 0 \\
0 & 0 & 1 \\
0 & 1 & 0
\end{array}\right) \\
& \lambda_{7}=\frac{1}{\sqrt{2}}\left(\begin{array}{ccc}
0 & 0 & 0 \\
0 & 0 & -i \\
0 & i & 0
\end{array}\right) ; \lambda_{8}=\frac{1}{\sqrt{6}}\left(\begin{array}{ccc}
1 & 0 & 0 \\
0 & 1 & 0 \\
0 & 0 & -2
\end{array}\right),
\end{aligned}
$$

which satisfy $\operatorname{Tr}\left[\lambda_{\alpha} \lambda_{\beta}\right]=\delta_{\alpha, \beta}$. In this basis, the computational spin-1 states are represented by the 3-dimensional vectors

$$
|1\rangle=\left(\begin{array}{l}
1 \\
0 \\
0
\end{array}\right) ;|0\rangle=\left(\begin{array}{l}
0 \\
1 \\
0
\end{array}\right) \text { and }|-1\rangle=\left(\begin{array}{l}
0 \\
0 \\
1
\end{array}\right) \text {. }
$$

Then, the relative purity for a generic pure state $|\psi\rangle$ becomes

$$
P_{\mathfrak{h}}(|\psi\rangle)=\frac{3}{4} \sum_{\alpha=1}^{8} \sum_{i=1}^{2}\left\langle\lambda_{\alpha}^{i}\right\rangle^{2},
$$

where $\left\langle\lambda_{\alpha}^{i}\right\rangle$ denotes the expectation value of $\lambda_{\alpha}^{i}$ in the state $|\psi\rangle$. In this way, product states like $|\psi\rangle=\left|\phi_{1}\right\rangle_{1} \otimes\left|\phi_{2}\right\rangle_{2}$ are generalized unentangled $\left(P_{\mathfrak{h}}=1\right.$ ) and states like $\left|\mathrm{GHZ}_{1}^{2}\right\rangle$ (and states connected through local spin unitary operations), are maximally entangled in this algebra $\left(P_{\mathfrak{h}}=0\right)$.

Different results are obtained if the purity is calculated relative to a subalgebra of local observables. For example, the two-spin-1 product state $|0,0\rangle=|0\rangle \otimes|0\rangle$ where both spins have zero projection along $z$ becomes generalized entangled relative to the (irreducible) algebra $\mathfrak{s u}(2)_{1} \oplus \mathfrak{s u}(2)_{2}$ of local spin rotations, which is generated by $\left\{S_{\alpha}^{i} ; i: 1,2 ; \alpha=x, y, z\right\}$, the spin-1 angular momentum operators $S_{\alpha}$ for each spin being given by

$$
S_{x}=\frac{1}{\sqrt{2}}\left(\begin{array}{ccc}
0 & 1 & 0 \\
1 & 0 & 1 \\
0 & 1 & 0
\end{array}\right), S_{y}=\frac{1}{\sqrt{2}}\left(\begin{array}{ccc}
0 & -i & 0 \\
i & 0 & -i \\
0 & i & 0
\end{array}\right), S_{z}=\left(\begin{array}{ccc}
1 & 0 & 0 \\
0 & 0 & 0 \\
0 & 0 & -1
\end{array}\right) \text {. }
$$


Notice that access to local angular momentum observables suffices to operationally characterize the system as describable in terms of two spin-1 particles (by imagining, for instance, performing a Stern-Gerlach-type of experiment on each particle). Thus, even when a subsystem decomposition can be naturally identified from the beginning in this case, states which are manifestly separable (unentangled) in the standard sense may exhibit GE (see also Appendix $\mathrm{A}$. On the other hand, this is physically quite natural in the example, since there are no $S U(2) \times S U(2)$ group operations (local rotations) that are able to transform the state $|0,0\rangle$ into the unentangled product state $|1,1\rangle$.

The examples described in this section together with other examples of states of bipartite quantum systems are shown in Fig. 1] It is clear that calculating the purity relative to different algebras gives information about different types of quantum correlations present in the system.

\section{B. $\quad N$-spin systems}

The traditional concept of pure multipartite entanglement in an $N$ spin- $S$ quantum system refers to quantum states that cannot be written as a product of states of each party. The $\mathfrak{h}$-purity distinguishes pure product states from entangled ones if it is calculated relative to the (irreducible)

algebra of local observables $\mathfrak{h}=\bigoplus_{i=1}^{N} \mathfrak{s u}(2 S+1)_{i}$ (see Appendix A). By Eq. (6), the measure $P_{\mathfrak{h}}$ is invariant under local unitary operations as desired. In particular, the usual concept of entanglement in an $N$-qubit quantum state ( $N$ spin $1 / 2 \mathrm{~s}$ ) can be recovered if the purity is calculated relative to the local algebra $\mathfrak{h}=\bigoplus_{i=1}^{N} \mathfrak{s u}(2)_{i}=\left\{\sigma_{x}^{1}, \sigma_{y}^{1}, \sigma_{z}^{1}, \cdots, \sigma_{x}^{N}, \sigma_{y}^{N}, \sigma_{z}^{N}\right\}$, where the Pauli operators $\sigma_{\alpha}^{i}$ $(\alpha=x, y, z)$ are now

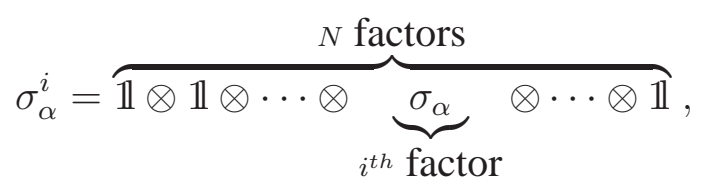

and the $2 \times 2$ matrices $\sigma_{\alpha}$ and $\mathbb{1}$ are given in Eq. (10). Then, the local purity becomes

$$
P_{\mathfrak{h}}(|\psi\rangle)=\frac{1}{N} \sum_{\alpha=x, y, z} \sum_{i=1}^{N}\left\langle\sigma_{\alpha}^{i}\right\rangle^{2}
$$

where again the normalization factor $1 / N$ is obtained by setting $P_{\mathfrak{h}}=1$ in any product state like $|\psi\rangle=\left|\phi_{1}\right\rangle_{1} \otimes\left|\phi_{2}\right\rangle_{2} \otimes \cdots \otimes\left|\phi_{N}\right\rangle_{N}$ (a GCS in this algebra). With this definition, states like $\left|\mathrm{GHZ}_{\frac{1}{2}}^{N}\right\rangle$, $[(|\uparrow, \downarrow\rangle-|\downarrow, \uparrow\rangle) / \sqrt{2}]^{\otimes n}$ (with obvious notations), and the cluster states $|\Phi\rangle_{C}$ introduced in Ref. [38] (see also Appendix [B] , will be maximally entangled $\left(P_{\mathfrak{h}}=0\right)$.

Remarkably, as announced in [11], after some algebraic manipulations (see Appendix [C), one 
can prove that

$$
P_{\mathfrak{h}}(|\psi\rangle)=1-Q(|\psi\rangle)
$$

where $Q$ is the (pure-state) measure of global entanglement for $N$ spin $1 / 2$ s systems originally introduced by Meyer and Wallach in [39]. A similar relation was independently derived in [40]. See also [41] for additional related considerations.

In Fig. 2 we display some examples of the purity relative to the local algebra $\mathfrak{h}=\bigoplus_{i=1}^{N} \mathfrak{s u}(2)_{i}$ for a $N$ spin- $S$ system. We also show the purity relative to the algebra of all observables $\mathfrak{s u}\left([2 S+1]^{N}\right)$, where any pure quantum state is a GCS, thus generalized unentangled $\left(P_{\mathfrak{h}}=1\right)$.

\section{Purity relative to the $\mathfrak{u}(N)$ algebra}

We now apply the concept of GE to a physical system consisting of $N$ (spinless) fermion modes $j$, each mode being described in terms of canonical creation and annihilation operators $c_{j}^{\dagger}$, $c_{j}$ respectively, satisfying the following anti-commutation rules:

$$
\left\{c_{i}^{\dagger}, c_{j}\right\}=\delta_{i, j}, \quad\left\{c_{i}, c_{j}\right\}=0 \text {. }
$$

For instance, different modes could be associated with different sites in a lattice, or to delocalized momentum modes related to the spatial modes through a Fourier transform. In general, for any $N \times N$ unitary matrix $U$, any transformation $c_{j} \mapsto \sum_{j} U_{i j} c_{j}$ maps the original modes into another possible set of fermionic modes. Using the above commutation relations, one also finds that

$$
\left[c_{i}^{\dagger} c_{j}, c_{k}^{\dagger} c_{l}\right]=\delta_{j k} c_{i}^{\dagger} c_{l}-\delta_{i l} c_{k}^{\dagger} c_{j}
$$

Thus, the set of bilinear fermionic operators $\left\{c_{j}^{\dagger} c_{j^{\prime}} ; 1 \leq j, j^{\prime} \leq N\right\}$ provides a realization of the unitary Lie algebra $\mathfrak{u}(N)$ in the $2^{N}$-dimensional Fock space $\mathcal{H}_{\text {Fock }}$ of the system. The latter is constructed as the direct sum of subspaces $\mathcal{H}_{n}$ corresponding to a fixed fermion number $n=$ $0, \ldots, N$, with $\operatorname{dim}\left(\mathcal{H}_{n}\right)=N ! /[n !(N-n) !]$. For our purposes, it is convenient to express $\mathfrak{u}(N)$ as the linear span of a Hermitian, orthonormal operator basis, which we choose as follows:

$$
\mathfrak{u}(N)=\left\{\begin{array}{cl}
\left(c_{j}^{\dagger} c_{j^{\prime}}+c_{j^{\prime}}^{\dagger} c_{j}\right) & \text { with } 1 \leq j<j^{\prime} \leq N \\
i\left(c_{j^{\prime}}^{\dagger} c_{j^{\prime}}-c_{j^{\prime}}^{\dagger} c_{j}\right) & \text { with } 1 \leq j<j^{\prime} \leq N \\
\sqrt{2}\left(c_{j}^{\dagger} c_{j}-1 / 2\right) & \text { with } 1 \leq j \leq N
\end{array},\right.
$$

(We use henceforth the notational convention that the large left curly bracket means "is the span of"). The action of $\mathfrak{u}(N)$ on $\mathcal{H}_{\text {Fock }}$ is reducible, because any operator in $\mathfrak{u}(N)$ conserves the 
total number of fermions $n=\left\langle\sum_{j=1}^{N} c_{j}^{\dagger} c_{j}\right\rangle$. It turns out that the irrep decomposition of $\mathfrak{u}(N)$ is identical to the direct sum into fixed-particle-number subspaces $\mathcal{H}_{n}$, each irrep thus appearing with multiplicity one.

Using Eq. (7), the $\mathfrak{h}$-purity of a generic pure many-fermion state relative to $\mathfrak{u}(N)$ becomes

$$
P_{\mathfrak{h}}(|\psi\rangle)=\frac{2}{N} \sum_{j<j^{\prime}=1}^{N}\left[\left\langle c_{j}^{\dagger} c_{j^{\prime}}+c_{j^{\prime}}^{\dagger} c_{j}\right\rangle^{2}-\left\langle c_{j}^{\dagger} c_{j^{\prime}}-c_{j^{\prime}}^{\dagger} c_{j}\right\rangle^{2}\right]+\frac{4}{N} \sum_{j=1}^{N}\left\langle c_{j}^{\dagger} c_{j}-1 / 2\right\rangle^{2} .
$$

Here, we took $\mathrm{K}=2 / N$, for reasons that will become clear shortly. In this case, the fermionic

\begin{tabular}{|c|c|c|}
\hline$\frac{1}{2}$ & $\mathfrak{s u}(2)_{1} \oplus \cdots \oplus \mathfrak{s u}(2)_{N}$ & $\mathfrak{s u}\left(2^{N}\right)$ \\
\hline & $\begin{array}{c}1 \longrightarrow\left|\mathrm{F}_{\frac{1}{2}}^{N}\right\rangle \\
{\left[\frac{N-2}{N}\right]^{2}----\left|\mathrm{W}_{\frac{1}{2}}^{N}\right\rangle} \\
\left.0 \longrightarrow \mathrm{GHZ}_{\frac{1}{2}}^{N}\right\rangle\end{array}$ & $1 \longrightarrow \begin{array}{l}\left|\mathrm{F}_{\frac{1}{2}}^{N}\right\rangle ;\left|\mathrm{W}_{\frac{1}{2}}^{N}\right\rangle ; \\
\left|\mathrm{GHZ}_{\frac{1}{2}}^{N}\right\rangle ; \cdots\end{array}$ \\
\hline 1 & $\mathfrak{s u}(2)_{1} \oplus \cdots \oplus \mathfrak{s u}(2)_{N}$ & $\mathfrak{s u}\left(3^{N}\right)$ \\
\hline & $\begin{array}{c}1 \longrightarrow\left|\mathrm{F}_{1}^{N}\right\rangle \\
{\left[\frac{N-1}{N}\right]^{2}----\left|\mathrm{W}_{1}^{N}\right\rangle} \\
\left.0 \longrightarrow \mathrm{GHZ}_{1}^{N}\right\rangle\end{array}$ & $1 \longrightarrow \begin{array}{l}\left|\mathrm{F}_{1}^{N}\right\rangle ;\left|\mathrm{W}_{1}^{N}\right\rangle ; \\
\left|\mathrm{GHZ}_{1}^{N}\right\rangle ; \cdots\end{array}$ \\
\hline$S$ & $\mathfrak{s u}(2)_{1} \oplus \cdots \oplus \mathfrak{s u}(2)_{N}$ & $\mathfrak{s u}\left([2 S+1]^{N}\right)$ \\
\hline & $\begin{array}{c}1 \\
{\left[\frac{N-\frac{1}{S}}{N}\right]^{2----}\left|\mathrm{W}_{S}^{N}\right\rangle} \\
\\
0 \\
\left|G H Z_{S}^{N}\right\rangle\end{array}$ & $\begin{array}{r}\left|\mathrm{F}_{S}^{N}\right\rangle ;\left|\mathrm{W}_{S}^{N}\right\rangle ; \\
\left|\mathrm{GHZ} Z_{S}^{N}\right\rangle ; \cdots\end{array}$ \\
\hline
\end{tabular}

FIG. 2: Purity relative to different algebras for a $N$ spin- $S$ system. The quantum states $\left|G H Z Z_{S}^{N}\right\rangle,\left|\mathrm{W}_{S}^{N}\right\rangle$, and $\left|\mathrm{F}_{S}^{N}\right\rangle$ are defined in Eqs. 9 . 
product states (Slater determinants) of the form $|\phi\rangle=\prod_{l} c_{l}^{\dagger}|\mathrm{vac}\rangle$, with $|\mathrm{vac}\rangle$ denoting the reference state with no fermions and $l$ labelling a particular set of modes, are the GCSs of the $\mathfrak{u}(N)$ algebra [32, 33]. Because a Slater determinant carries a well defined number of particles, each GCS belongs to an irrep space $\mathcal{H}_{n}$ for some $n$, states with different $n$ belonging to different orbits under $\mathfrak{u}(N)$. A fixed GCS has maximum $\mathfrak{h}$-purity when compared to any other state within the same irrep space. Remarkably, it also turns out that any GCS of $\mathfrak{h}=\mathfrak{u}(N)$ gives rise to a reduced state which is extremal (thus generalized unentangled) regardless of $n$, the $\mathfrak{h}$-purity assuming the same (maximum) value in each irrep. Using this property, the normalization factor $\mathrm{K}=2 / N$ was calculated by setting $P_{\mathfrak{h}}=1$ in an arbitrary Slater determinant. Thus, the purity relative to the $\mathfrak{u}(N)$ algebra is a good measure of entanglement in fermionic systems, in the sense that $P_{\mathfrak{h}}=1$ in any fermionic product state, and $P_{\mathfrak{h}}<1$ for any other state, irrespective of whether the latter has a well defined number of fermions or not. Notice that, thanks to the invariance of $P_{\mathfrak{h}}$ under group transformations (Eq. (6)), the property of a state being generalized unentangled is independent of the specific set of modes that is chosen. This is an important difference between our GE and the mode entanglement approach [26, 42].

\section{ENTANGLEMENT AND QUANTUM PHASE TRANSITIONS}

As already mentioned, although many measures of entanglement have been defined in the literature, assessing their ability to help us better understand QPTs in quantum systems largely remains an open problem. In the following two sections we attempt to characterize the QPTs present in the LMG model and in the anisotropic XY model in an external magnetic field through the GE notion, relative to a particular subset of observables which will be appropriately chosen in each case. Interestingly, for both these models the ground states can be computed exactly by mapping the set of observable operators involved in the system Hamiltonian to a new set of operators which satisfy the same commutation relations, thus preserving the underlying algebraic structure. In the new operator language, the models are seen to contain some symmetries that make them exactly solvable, allowing one to obtain the ground state properties in a number of operations that scales polynomially with the system size (see also [43] for related discussions). It is possible then to understand which quantum correlations give rise to the QPTs in these cases.

Several issues should be considered when looking for an algebra $\mathfrak{h}$ of observables that may make the corresponding relative purity a good indicator of a QPT. A first relevant observation is 
that in each of these cases a preferred Lie algebra exists, where the respective ground state would have maximum $\mathfrak{h}$-purity independently of the interaction strengths in the Hamiltonian. The purity relative to such an algebra remains constant, therefore it does not identify the QPT. (In these cases, this algebra is in fact the Lie algebra generated by the parametrized family of model Hamiltonians, as the parameters are varied.) Thus, one needs to extract a subalgebra relative to which the ground state may be generalized entangled, depending on the parameters in the Hamiltonian. A second, closely related observation is that the purity must contain information about quantum correlations which undergo a qualitative change as the transition point is crossed: thus, the corresponding degree of entanglement, as measured by the purity, must depend on the interaction strengths governing the phase transition. Finally, whenever a degeneracy of the ground state exists or emerges in the thermodynamic limit, a physical requirement is that the purity be the same for all ground states.

Although these restrictions together turn out to be sufficient for choosing the relevant algebra of observables in the following two models, they do not provide an unambiguous answer when solving a non-integrable model whose exact ground state solution cannot be computed efficiently. Typically, in the latter cases the ground states are GCSs of Lie algebras each of whose dimension increases exponentially with the system size. Choosing the observable subalgebra that contains the proper information on the QPTs (such as information on critical exponents) then becomes, in general, a difficult task.

On the other hand, a concept of generalized mean-field Hamiltonian emerges from these considerations. Given a Hilbert space $\mathcal{H}$ of dimension $p^{N}$ (with $p$ an integer $>1$ ), we will define a mean-field Hamiltonian as an operator

$$
H_{\mathrm{MF}}=\sum_{\alpha} \epsilon_{\alpha} A_{\alpha}, \quad \epsilon_{\alpha} \in \mathbb{R}
$$

that is an element of an irreducibly represented Lie algebra of Hermitian operators $\mathfrak{h}=$ $\left\{A_{1}, \cdots, A_{L}\right\}$ whose dimension scales polynomially in $N$ that is, $L=\operatorname{poly}(N)$. When the ground state of such an $H_{\mathrm{MF}}$ is non-degenerate, it turns out to be a GCS of $\mathfrak{h}$ [15], while the remaining eigenstates (some of which may also be GCSs) and energies can be efficiently computed. The connection between Lie-algebraic mean-field Hamiltonians and their efficient solvability deserves a careful analysis in its own right, which we will present elsewhere [44]. 


\section{LIPKIN-MESHKOV-GLICK MODEL}

Originally introduced in the context of nuclear physics [21], the Lipkin-Meshov-Glick (LMG) model is widely used as a testbed for studying critical phenomena in (pseudo)spin systems [32]. This model was shown to be exactly-solvable in [22]. In this section, we investigate the critical properties of this model by calculating the purity relative to a particular subset of observables, which will be chosen by analyzing the classical behavior of the ground state of the system. For this purpose, we first need to map the model to a single spin, where it becomes solvable and where the standard notion of entanglement is not immediately applicable.

The model is constructed by considering $N$ fermions distributed in two $N$-fold degenerate levels (termed upper and lower shells). The latter are separated by an energy gap $\epsilon$, which will be set here equal to 1 . The quantum number $\sigma= \pm 1$ ( $\uparrow$ or $\downarrow$ ) labels the level while the quantum number $k$ denotes the particular degenerate state in the level (for both shells, $k \in\left\{k_{1}, \ldots, k_{N}\right\}$ ). In addition, we consider a "monopole-monopole" interaction that scatters pairs of particles between the two levels without changing $k$. The model Hamiltonian may be written as

$$
H=H_{0}+\hat{V}+\hat{W}=\frac{1}{2} \sum_{k, \sigma} \sigma c_{k \sigma}^{\dagger} c_{k \sigma}+\frac{V}{2 N} \sum_{k, k^{\prime}, \sigma} c_{k \sigma}^{\dagger} c_{k^{\prime} \sigma}^{\dagger} c_{k^{\prime} \bar{\sigma}} c_{k \bar{\sigma}}+\frac{W}{2 N} \sum_{k, k^{\prime}, \sigma} c_{k \sigma}^{\dagger} c_{k^{\prime} \bar{\sigma}}^{\dagger} c_{k^{\prime} \sigma} c_{k \bar{\sigma}},
$$

where $\bar{\sigma}=-\sigma$, and the fermionic operators $c_{k \sigma}^{\dagger}\left(c_{k \sigma}\right)$ create (annihilate) a fermion in the level identified by the quantum numbers $(k, \sigma)$ and satisfy the fermionic commutation relations given in Section $\amalg \mathrm{C}$ Thus, the interaction $\hat{V}$ scatters a pair of particles belonging to one of the levels, and the interaction $\hat{W}$ scatters a pair of particles belonging to different levels. Note that the factor $1 / N$ must be present in the interaction terms for stability reasons, as the energy per particle must be finite in the thermodynamic limit.

Upon introducing the pseudospin operators

$$
\begin{aligned}
& J_{+}=\sum_{k} c_{k \uparrow}^{\dagger} c_{k \downarrow}, \\
& J_{-}=\sum_{k} c_{k \downarrow}^{\dagger} c_{k \uparrow}, \\
& J_{z}=\frac{1}{2} \sum_{k, \sigma} \sigma c_{k \sigma}^{\dagger} c_{k \sigma}=\frac{1}{2}\left(n_{\uparrow}-n_{\downarrow}\right),
\end{aligned}
$$

which satisfy the $\mathfrak{s u}(2)$ commutation relations of the angular momentum algebra,

$$
\begin{aligned}
& {\left[J_{z}, J_{ \pm}\right]= \pm J_{ \pm},} \\
& {\left[J_{+}, J_{-}\right]=J_{z},}
\end{aligned}
$$


the Hamiltonian of Eq. (23) may be rewritten as

$$
H=J_{z}+\frac{V}{2 N}\left(J_{+}^{2}+J_{-}^{2}\right)+\frac{W}{2 N}\left(J_{+} J_{-}+J_{-} J_{+}\right) .
$$

As defined by Eq. (29), $H$ is invariant under the $\mathbb{Z}_{2}$ inversion symmetry operation $K$ that transforms $\left(J_{x}, J_{y}, J_{z}\right) \mapsto\left(-J_{x},-J_{y}, J_{z}\right)$, and it also commutes with the (Casimir) total angular momentum operator $\mathbf{J}^{2}=J_{x}^{2}+J_{y}^{2}+J_{z}^{2}$. Therefore, the non-degenerate eigenstates of $H$ are simultaneous eigenstates of both $K$ and $\mathbf{J}^{2}$, and they may be obtained by diagonalizing matrices of dimension $2 J+1$ (whereby the solubility of the model). Notice that, by definition of $J_{z}$ as in Eq. (26), the maximum eigenvalue of $J_{z}$ and $J=|\mathbf{J}|$ is $N / 2$. In particular, for a system with $N$ fermions as assumed, both the ground state $|g\rangle$ and first excited state $|e\rangle$ belong to the largest possible angular momentum eigenvalue $J=N / 2[21]$ (so-called half-filling configurations); thus, they can be computed by diagonalizing a matrix of dimension $N+1$.

The Hamiltonian (29) does not exhibit a QPT for finite $N$. It is important to remark that some critical properties of the LMG model in the thermodynamic limit $N \rightarrow \infty$ can be understood by using a semiclassical approach [45] (note that the critical behavior is essentially mean-field): first, we replace the angular momentum operators in $H / N$ (with $H$ given in Eq.(29) by their classical components (Fig. 3); that is

$$
\begin{aligned}
\mathbf{J}=\left(J_{x}, J_{y}, J_{z}\right) & \rightarrow(J \sin \theta \cos \phi, J \sin \theta \sin \phi, J \cos \theta), \\
H / N & \rightarrow h_{c}(j, \theta, \phi),
\end{aligned}
$$

where $h_{c}$ is the resulting classical Hamiltonian and $j=J / N, j=0, \ldots, 1 / 2$. In this way, one can show that in the thermodynamic limit (see Appendix D)

$$
\lim _{N \rightarrow \infty} \frac{\langle g|H| g\rangle}{N}=\lim _{N \rightarrow \infty} \frac{E_{g}}{N}=\min _{j, \theta, \phi} h_{c}(j, \theta, \phi),
$$

so the ground state energy per particle $E_{g} / N$ can be easily evaluated by minimizing

$$
h_{c}(j, \theta, \phi)=j \cos \theta+\frac{V}{2} j^{2} \sin ^{2} \theta \cos (2 \phi)+W j^{2} \sin ^{2} \theta .
$$

As mentioned, the ground and first excited states have maximum angular momentum $j=1 / 2$. In Fig. 4 we show the orientation of the angular momentum in the ground states of the classical Hamiltonian $h_{c}$, represented by the vectors $\mathbf{J}, \mathbf{J}_{1}$, and $\mathbf{J}_{2}$, for different values of $V$ and $W$. When $\Delta=|V|-W \leq 1$ we have $\theta=\pi$ and the classical angular momentum is oriented in the negative $z$-direction. However, when $\Delta>1$ we have $\cos \theta=-\Delta^{-1}$ and the classical ground state becomes 


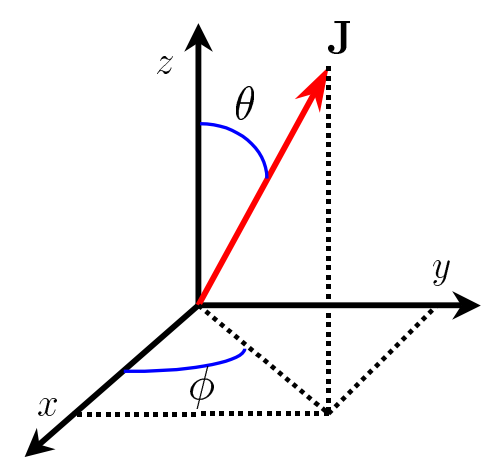

FIG. 3: Angular momentum coordinates in the three-dimensional space.

two-fold degenerate (notice that $h_{c}$ is invariant under the transformation $\phi \mapsto-\phi$ ). In this region and for $V<0$ the angular momentum is oriented in the $x z$ plane $(\phi=0)$ while for $V>0$ it is oriented in the $y z$ plane $(\phi= \pm \pi / 2)$. The model has a gauge symmetry in the line $V=0$, $W<-1$, where $\phi$ can take any possible value.

\section{A. First and second order QPTs, and critical behavior}

Going back to the original Hamiltonian of Eq. (23), the quantum system undergoes a second order QPT at the critical boundary $\Delta_{c}=\left|V_{c}\right|-W_{c}=1$, where for $\Delta>\Delta_{c}$ the ground and first excited states $|g\rangle$ and $|e\rangle$ become degenerate in the thermodynamic limit and the inversion symmetry $K$ breaks. The order parameter is given by the mean number of fermions in the upper shell $\left\langle n_{\uparrow}\right\rangle=1 / 2+\left\langle J_{z}\right\rangle / N$, which in the thermodynamic limit converges to its classical value,

$$
\lim _{N \rightarrow \infty}\left\langle n_{\uparrow}\right\rangle=\frac{1+\cos \theta}{2} .
$$

Obviously, for $\Delta \leq \Delta_{c}$ we have $\left\langle n_{\uparrow}\right\rangle=0$, and $\left\langle n_{\uparrow}\right\rangle>0$ otherwise (see Fig 4). The critical exponents of the order parameter are easily computed by making a Taylor expansion near the critical points $\left(\Delta \rightarrow 1^{+}\right)$. Defining the quantities $x=V_{c}-V$ and $y=W_{c}-W$, we obtain

$$
\lim _{\Delta \rightarrow 1^{+}}\left\langle n_{\uparrow}\right\rangle=\left\{\begin{array}{ll}
\left(y^{\alpha}-x^{\beta}\right) / 2 & \text { for } V>0 \\
\left(y^{\alpha}+x^{\beta}\right) / 2 & \text { for } V<0
\end{array},\right.
$$

where the critical exponents are $\alpha=1$ and $\beta=1$.

In Fig. 5 we show the exact ground state energy per particle $E_{g} / N$ (with $E_{g}=\langle g|H| g\rangle$ ) as a function of $V$ and $W$ in the thermodynamic limit (Eqs. (32)). One can see that also in the broken 


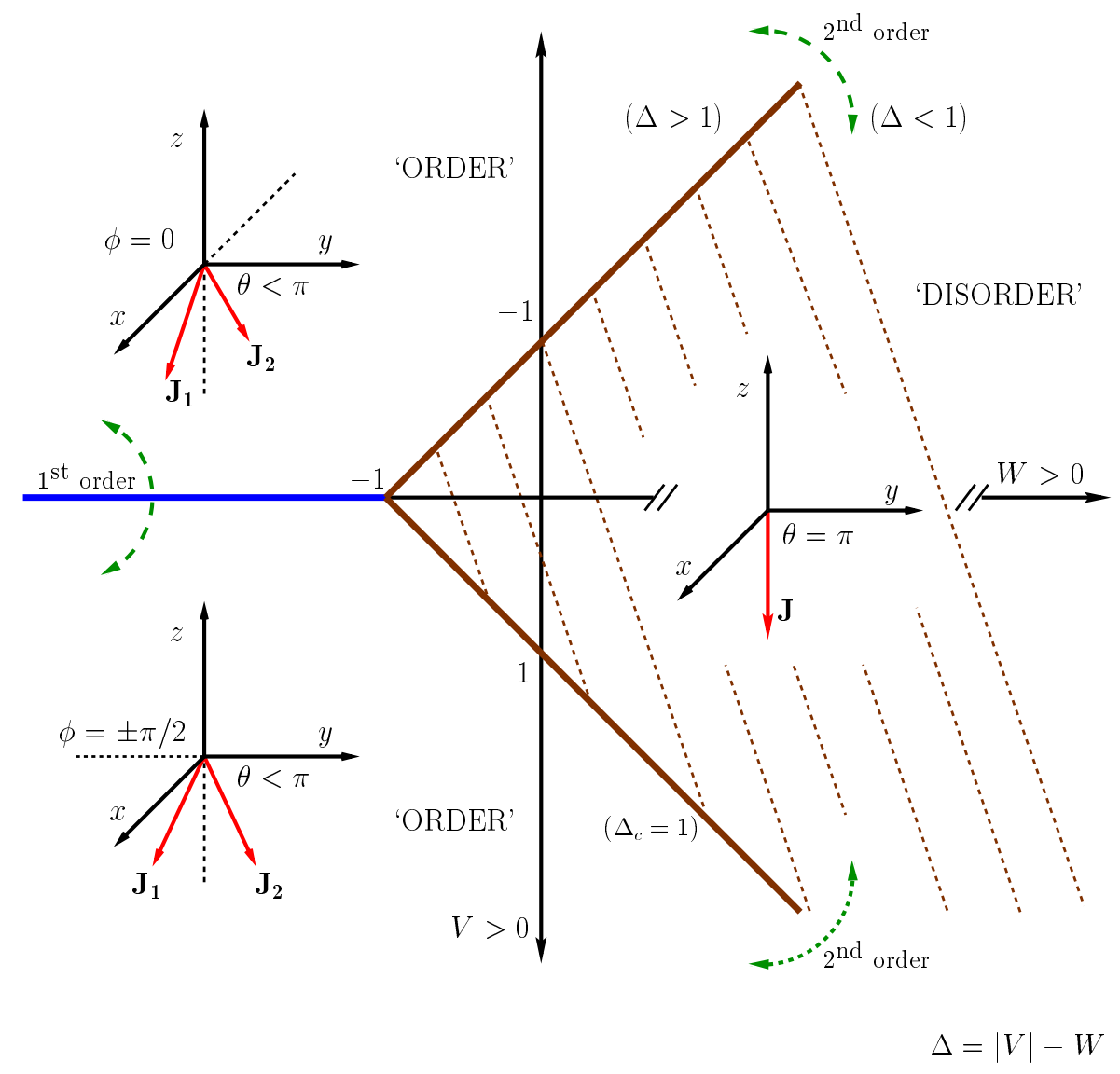

FIG. 4: Representation of the classical ground state of the LMG model.

symmetry region $(\Delta>1)$ the system undergoes a first order QPT at $V=0$; that is, the first derivative of the ground state energy with respect to $V$ is not continuous in this line.

\section{B. Purity as an indicator of the QPTs in the LMG model}

The standard notion of entanglement is not directly applicable to the LMG model as described by Eq. (29), for this is a single spin system and no physically natural partition into subsystems is possible. Therefore, using the $\mathfrak{h}$-purity as a measure of entanglement becomes an advantage from this point of view, since the latter only depends on a particular subset of observables and no partition of the system is necessary. The first required step is the identification of a relevant Lie algebra of observables relative to which the purity has to be calculated.

Since both the ground and first excited states of the quantum LMG model may be understood as states of a system carrying total angular momentum $J=N / 2$, a first possible algebra to consider 


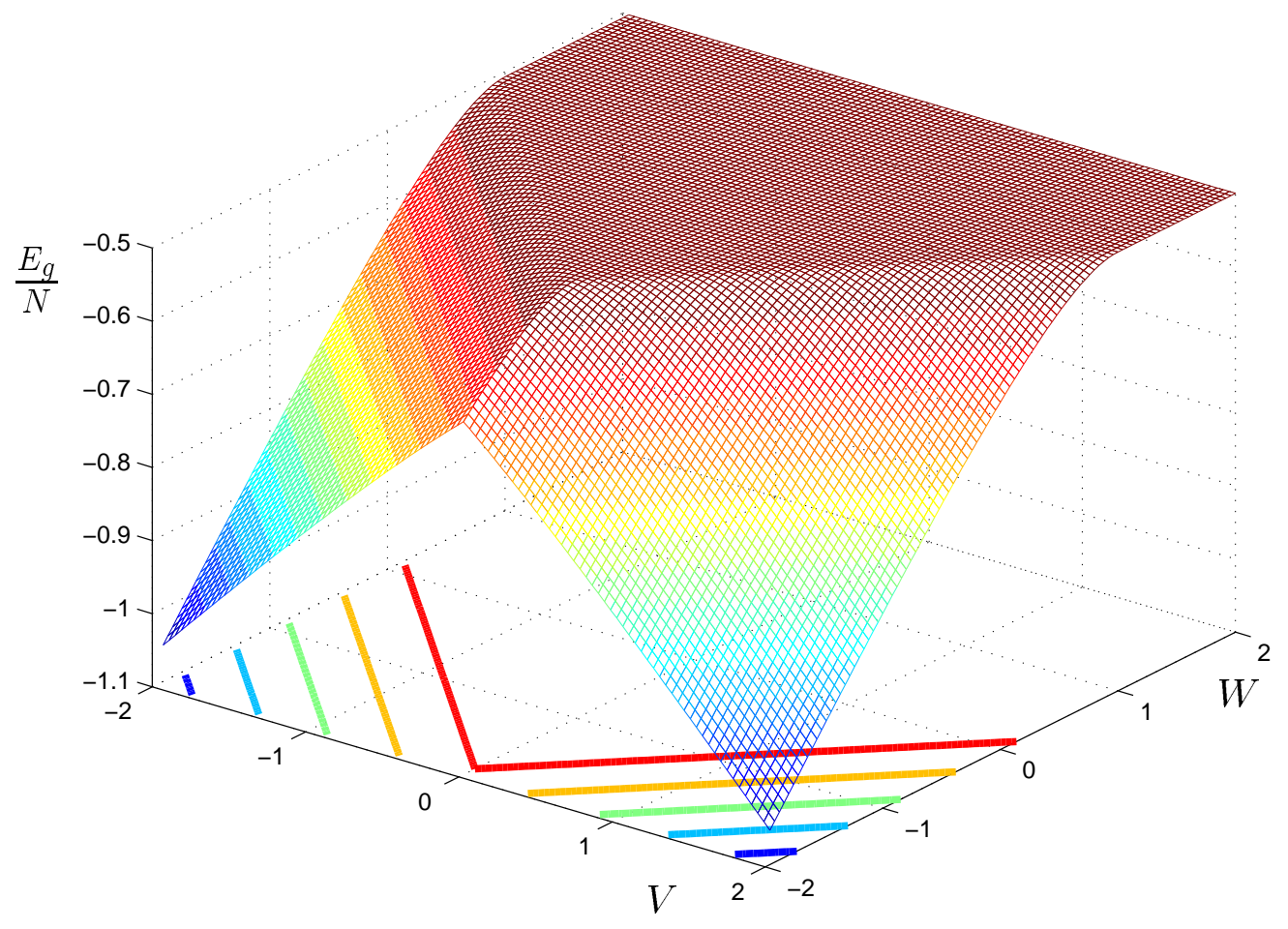

FIG. 5: Ground state energy per particle in the LMG model.

is the $\mathfrak{s u}(N+1)$ algebra acting on the relevant $(N+1)$-dimensional eigenspace. Relative to this algebra, $|g\rangle$ is generalized unentangled for arbitrary values of $V, W$ thus the corresponding purity remains constant and does not signal the QPTs. However, the family of Hamiltonians (29) do not generate this Lie algebra, but rather an $\mathfrak{s u}(2)$ algebra, so perhaps $\mathfrak{s u}(N+1)$ is not a natural choice physically [46].

Thus a natural choice, suggested by the commutation relationships of Eqs. (27) and (28), is to study the purity relative to the spin- $N / 2$ representation of the angular momentum Lie algebra $\mathfrak{h}=\mathfrak{s u}(2)=\left\{J_{x}, J_{y}, J_{z}\right\}:$

$$
P_{\mathfrak{h}}(|\psi\rangle)=\frac{4}{N^{2}}\left[\left\langle J_{x}\right\rangle^{2}+\left\langle J_{y}\right\rangle^{2}+\left\langle J_{z}\right\rangle^{2}\right],
$$

where the normalization factor $\mathrm{K}=N^{2} / 4$ is chosen to ensure that the maximum of $P_{\mathfrak{h}}$ is equal to 1 . With this normalization factor, $P_{\mathfrak{h}}$ can be calculated exactly in the thermodynamic limit by relying on the semi-classical approach described earlier (see Appendix D and Eq. (30)). For $V=0$ and arbitrary $W>0,|g\rangle=\left|J_{z}=-N / 2\right\rangle$ which is a GCS of $\mathfrak{s u}(2)$ and has $P_{\mathfrak{h}}=1$. For generic interaction values such that $\Delta \leq 1$, the classical angular momentum depicted in Fig. 
4 is oriented along the $z$-direction and is not degenerate: because $\left\langle J_{x}\right\rangle=\left\langle J_{y}\right\rangle=0$, only $\left\langle J_{z}\right\rangle$ contributes to $P_{\mathfrak{h}}$; by recalling that $\lim _{N \rightarrow \infty}\left\langle J_{z} / N\right\rangle=-1 / 2$, this gives $P_{\mathfrak{h}}=1$, so that as far as relative purity is concerned the ground state behaves asymptotically like a coherent state in the thermodynamic limit. Physically, this means that with respect to the relevant fluctuations, GCSs of $\mathfrak{s u}(2)$ are a good approximation of the quantum ground state for large particle numbers, as is well established for this model [47]. However, in the region $\Delta>1$ the ground state (both classical and quantum) is two-fold degenerate in the $N \rightarrow \infty$ limit, and the value of $P_{\mathfrak{h}}$ depends in general on the particular linear combination of degenerate states. This can be understood from Fig. 4 where different linear combinations of the two degenerate vectors $\mathbf{J}_{1}$ and $\mathbf{J}_{2}$ imply different values of $\left\langle J_{x}\right\rangle$ for $V<0$ and different values of $\left\langle J_{y}\right\rangle$ for $V>0$, while $\left\langle J_{z}\right\rangle$ remains constant. With these features, the purity relative to the $\mathfrak{s u}(2)$ algebra will not be a good indicator of the QPT.

An alternative option is then to look at a subalgebra of $\mathfrak{s u}(2)$. In particular, if we only consider the purity relative to the single observable $\mathfrak{h}=\mathfrak{s o}(2)=\left\{J_{z}\right\}$ (i.e., a particular CSA of $\mathfrak{s u}(2)$ ), and retain the same normalization as above, we have

$$
P_{\mathfrak{h}}(|\psi\rangle)=\frac{4}{N^{2}}\left\langle J_{z}\right\rangle^{2}
$$

This new purity will be a good indicator of the QPT, since $P_{\mathfrak{h}}=1$ only for $\Delta \leq 1$ in the thermodynamic limit, and in addition $P_{\mathfrak{h}}$ does not depend on the particular linear combination of the two-fold degenerate states in the region $\Delta>1$, where $P_{\mathfrak{h}}<1$. Obviously, in this case $P_{\mathfrak{h}}$ is straightforwardly related to the order parameter (Eq. (34)); the critical exponents of $P_{\mathfrak{h}}-1$ are indeed the same $(\alpha=1$ and $\beta=1)$.

Note that the purity defined by Eq. (36) does not always take its maximum value for GCSs of $\mathfrak{h}=\mathfrak{s o}(2)$ (eigenstates of $J_{z}$ ). In the region $\Delta<1$ where $P_{\mathfrak{h}}=1$, the quantum ground state of the LMG model (Eq. (29) does not have a well defined $z$-component of angular momentum except at $V=0\left(\left[H, J_{z}\right] \neq 0\right.$ if $\left.V \neq 0\right)$, thus in general it does not lie on a coherent orbit of this algebra for finite $N$. However, as discussed above, it behaves asymptotically (in the infinite $N$ limit) as a GCS (in the sense that $P_{\mathfrak{h}} \rightarrow 1$ ). Moreover, in Section $\amalg$ we showed that for $J_{z}$-eigenstates with eigenvalues $\left|J_{z}\right|<N / 2$, we also obtain $P_{\mathfrak{h}}<1$.

In Fig. 6 we show the behavior of $P_{\mathfrak{h}}$ as a function of the parameters $V$ and $W$. Interestingly, the purity relative to $J_{z}$ is a good indicator not only of the second order QPT but also of the first order QPT (the line $V=0, W<-1$ ). 


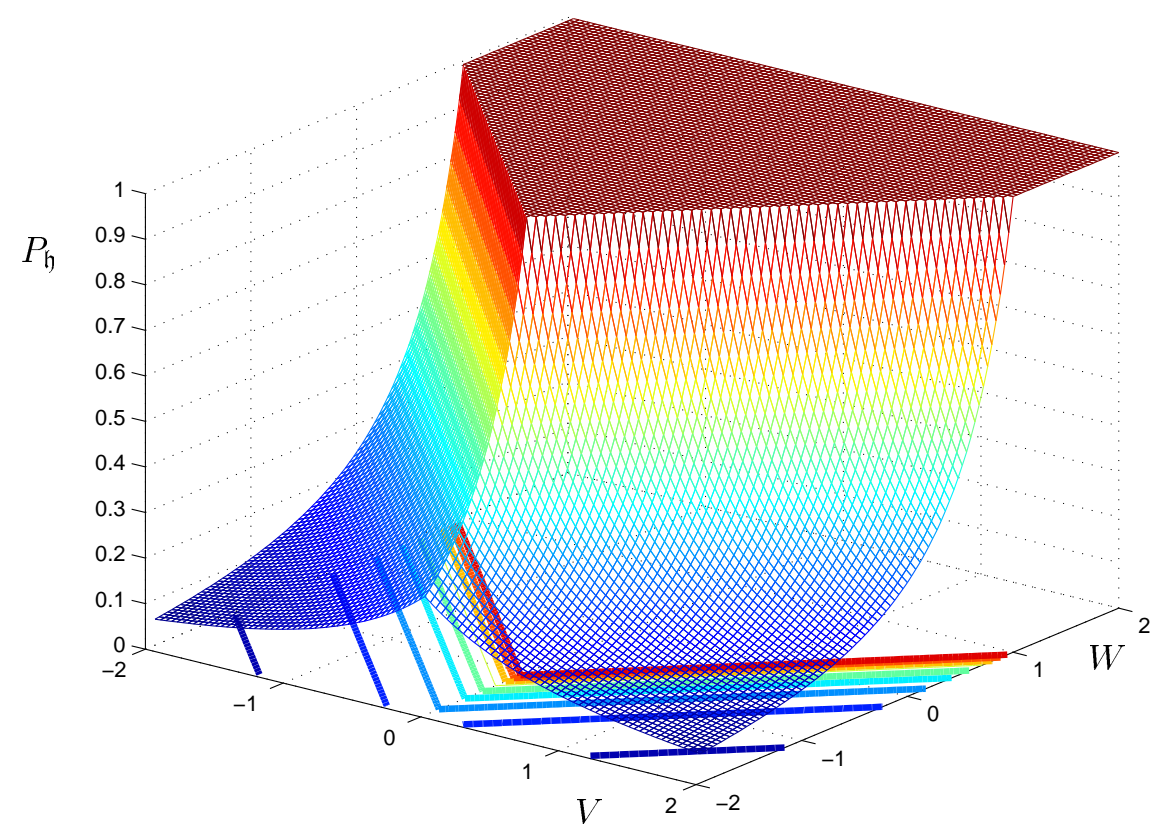

FIG. 6: Purity relative to the observable $J_{z}$ in the ground state of the LMG model.

\section{ANISOTROPIC XY MODEL IN A TRANSVERSE MAGNETIC FIELD}

In this section, we exploit the purity relative to the $\mathfrak{u}(N)$ algebra (introduced in Section (世C) ) as a measure able to identify the paramagnetic to ferromagnetic QPT in the anisotropic onedimensional spin-1/2 XY model in a transverse magnetic field and classify its universality properties.

The model Hamiltonian for a chain of $N$ sites is given by (see Fig (7)

$$
H=-g \sum_{i=1}^{N}\left[(1+\gamma) \sigma_{x}^{i} \sigma_{x}^{i+1}+(1-\gamma) \sigma_{y}^{i} \sigma_{y}^{i+1}\right]+\sum_{i=1}^{N} \sigma_{z}^{i}
$$

where the operators $\sigma_{\alpha}^{i}(\alpha=x, y, z)$ are the Pauli spin-1/2 operators on site $i$ (defined in Eqs. (10) and (15)), $g$ is the parameter one may tune to drive the QPT, and $0<\gamma \leq 1$ is the amount of anisotropy in the $x y$ plane. In particular, for $\gamma=1 \mathrm{Eq}$. (37) reduces to the Ising model in a transverse magnetic field, while for $\gamma \rightarrow 0$ the model becomes isotropic. Periodic boundary conditions were considered here, that is $\sigma_{\alpha}^{i+N}=\sigma_{\alpha}^{i}$, for all $i$ and $\alpha$.

When $g \gg 1$ and $\gamma=1$ the model is Ising-like. In this limit, the spin-spin interactions are the dominant contribution to the Hamiltonian [37, and the ground state becomes degenerate in the thermodynamic limit, exhibiting ferromagnetic long-range order correlations in the $x$ direction: 


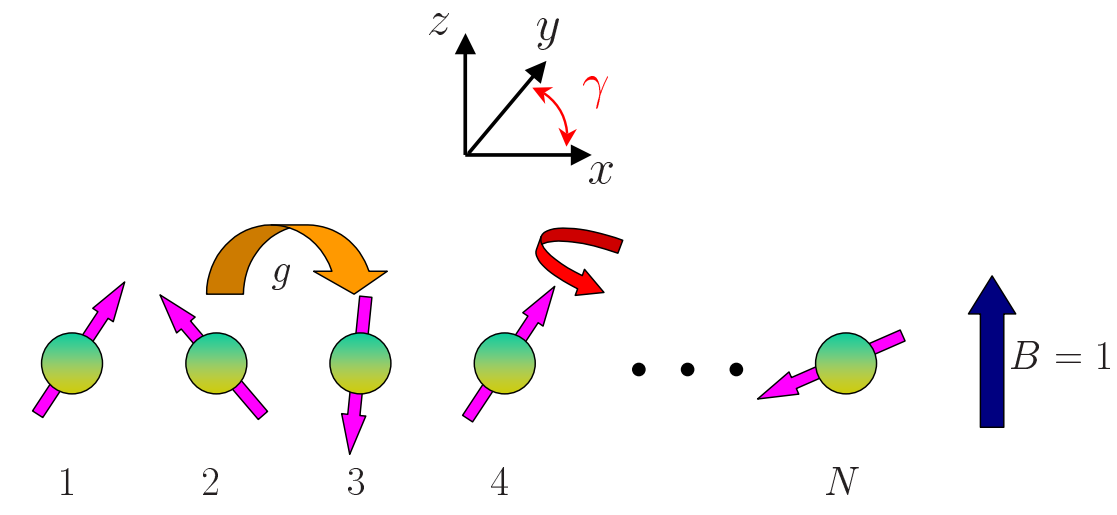

FIG. 7: Anisotropic one-dimensional XY model in an external transverse magnetic field $B$.

$M_{x}^{2}=\lim _{N \rightarrow \infty}\left\langle\sigma_{x}^{1} \sigma_{x}^{N / 2}\right\rangle>0$, where $M_{x}$ is the magnetization in the $x$-direction. In the opposite limit where $g \rightarrow 0$, the external magnetic field becomes important, the spins tend to align in the $z$ direction, and the magnetization in the $x$ direction vanishes: $M_{x}^{2}=\lim _{N \rightarrow \infty}\left\langle\sigma_{x}^{1} \sigma_{x}^{N / 2}\right\rangle=0$. Thus, in the thermodynamic limit the model is subject to a paramagnetic-to-ferromagnetic second order QPT at a critical point $g_{c}$ that will be determined later, with critical behavior belonging to the 2- $D$ Ising model universality class.

This model can be exactly solved using the Jordan-Wigner transformation [48], which maps the Pauli (spin 1/2) algebra into the canonical fermion algebra through

$$
c_{j}^{\dagger}=\prod_{l=1}^{j-1}\left(-\sigma_{z}^{l}\right) \sigma_{+}^{j},
$$

where the fermionic operators $c_{j}^{\dagger}\left(c_{j}\right)$ have been introduced in Section $\amalg \mathrm{Cl}$ and $\sigma_{+}^{j}=\left(\sigma_{x}^{j}+i \sigma_{y}^{j}\right) / 2$ is the raising spin operator.

In order to find the exact ground state, we first need to write the Hamiltonian given in Eq. 37] in terms of these fermionic operators,

$$
H=-2 g \sum_{i=1}^{N-1}\left(c_{i}^{\dagger} c_{i+1}+\gamma c_{i}^{\dagger} c_{i+1}^{\dagger}+\text { h.c. }\right)+2 g K\left(c_{N}^{\dagger} c_{1}+\gamma c_{N}^{\dagger} c_{1}^{\dagger}+\text { h.c. }\right)+2 \hat{N}
$$

where $K=\prod_{j=1}^{N}\left(-\sigma_{z}^{j}\right)$ is an operator that commutes with the Hamiltonian, and $\hat{N}=\sum_{i=1}^{N} c_{i}^{\dagger} c_{i}$ is the total number operator (here, we choose $N$ to be even). Then, the eigenvalue of $K$ is a good quantum number, and noticing that $K=e^{i \pi \hat{N}}$ we obtain $K=+1(-1)$ whenever the (non-degenerate) 
eigenstate of $H$ is a linear combination of states with an even (odd) number of fermions. In particular, the numerical solution of this model in finite systems (with $N$ even) indicates that the ground state has eigenvalue $K=+1$, implying anti-periodic boundary conditions in Eq. (39).

The second step is to re-write the Hamiltonian in terms of the fermionic operators $\tilde{c}_{k}^{\dagger}\left(\tilde{c}_{k}\right)$, defined by the Fourier transform of the operators $c_{j}^{\dagger}\left(c_{j}\right)$ :

$$
\tilde{c}_{k}^{\dagger}=\frac{1}{\sqrt{N}} \sum_{j=1}^{N} e^{-i k j} c_{j}^{\dagger}
$$

where the set $V$ of possible $k$ is determined by the anti-periodic boundary conditions in the fermionic operators: $V=V_{+}+V_{-}=\left[ \pm \frac{\pi}{N}, \pm \frac{3 \pi}{N}, \cdots, \pm \frac{(N-1) \pi}{N}\right]$. Therefore, we rewrite the Hamiltonian as

$$
H+N=-2 \sum_{k \in V}(-1+2 g \cos k) \tilde{c}_{k}^{\dagger} \tilde{c}_{k}+i g \gamma \sin k\left(\tilde{c}_{-k}^{\dagger} \tilde{c}_{k}^{\dagger}+\tilde{c}_{-k} \tilde{c}_{k}\right) .
$$

The third and final step is to diagonalize the Hamiltonian of Eq. (41) using the Bogoliubov canonical transformation

$$
\left\{\begin{array}{c}
\gamma_{k}=u_{k} \tilde{c}_{k}-i v_{k} \tilde{c}_{-k}^{\dagger} \\
\gamma_{-k}^{\dagger}=u_{k} \tilde{c}_{-k}^{\dagger}-i v_{k} \tilde{c}_{k}
\end{array},\right.
$$

where the real coefficients $u_{k}$ and $v_{k}$ satisfy the relations

$$
u_{k}=u_{-k}, v_{k}=-v_{-k}, \text { and } u_{k}^{2}+v_{k}^{2}=1,
$$

where

$$
u_{k}=\cos \left(\frac{\phi_{k}}{2}\right), v_{k}=\sin \left(\frac{\phi_{k}}{2}\right)
$$

with $\phi_{k}$ given by

$$
\tan \left(\phi_{k}\right)=\frac{2 g \gamma \sin k}{-1+2 g \cos k}
$$

In this way, the quasiparticle creation and annihilation operators $\gamma_{k}^{\dagger}$ and $\gamma_{k}$, satisfy the canonical fermionic anti-commutation relations of Eq. (18), and the Hamiltonian may be finally rewritten as

$$
H=\sum_{k \in V} \xi_{k}\left(\gamma_{k}^{\dagger} \gamma_{k}-1 / 2\right)
$$

where $\xi_{k}=2 \sqrt{(-1+2 g \cos k)^{2}+4 g^{2} \gamma^{2} \sin ^{2} k}$ is the quasiparticle energy. Since in general $\xi_{k}>0$, the ground state is the quantum state with no quasiparticles (BCS state [49]), such that $\gamma_{k}|\mathrm{BCS}\rangle=0$. Thus, one finds

$$
|\mathrm{BCS}\rangle=\prod_{k \in V_{+}}\left(u_{k}+i v_{k} \tilde{c}_{k}^{\dagger} \tilde{c}_{-k}^{\dagger}\right)|\mathrm{vac}\rangle
$$


where $|\mathrm{vac}\rangle$ is the state with no fermions $\left(\tilde{c}_{k}|\mathrm{vac}\rangle=0\right)$.

Excited states with an even number of fermions $(K=+1)$ can be obtained applying pairs of quasiparticle creation operators $\gamma_{k}^{\dagger}$ to the $|\mathrm{BCS}\rangle$ state. However, one should be more rigorous when obtaining excited states with an odd number of particles, since $K=-1$ implies periodic boundary conditions in Eq. (39), and the new set of possible $k$ 's (wave vectors) is $\bar{V}=\left[-\pi, \cdots,-\frac{2 \pi}{N}, 0, \frac{2 \pi}{N}, \cdots, \frac{2(N-1) \pi}{N}\right]$ (different of $\left.V\right)$.

\section{A. QPT and critical point}

In Fig. 8 we show the order parameter $M_{x}^{2}=\lim _{N \rightarrow \infty}\left\langle\sigma_{x}^{1} \sigma_{x}^{N / 2}\right\rangle$ as a function of $g$ in the thermodynamic limit and for different anisotropies $\gamma$ [4]. We observe that $M_{x}^{2}=0$ for $g \leq g_{c}$ and $M_{x}^{2} \neq 0$ for $g>g_{c}$, so the critical point is located at $g_{c}=1 / 2$, regardless of the value of $\gamma$. The value of $g_{c}$ can also be obtained by setting $\xi_{k}=0$ in Eq. [45], where the gap vanishes.

Notice that the Hamiltonian of Eq. 37 is invariant under the transformation that maps $\left(\sigma_{x}^{i} ; \sigma_{y}^{j} ; \sigma_{z}^{k}\right) \mapsto\left(-\sigma_{x}^{i} ;-\sigma_{y}^{j} ; \sigma_{z}^{k}\right)\left(\mathbb{Z}_{2}\right.$ symmetry $)$, implying that $\left\langle\sigma_{x}^{i}\right\rangle=0$ for all $g$. However, since in the thermodynamic limit the ground state becomes two-fold degenerate, for $g>g_{c}$, it is possible to build up a ground state where the discrete $\mathbb{Z}_{2}$ symmetry is broken, i.e. $\left\langle\sigma_{x}^{i}\right\rangle \neq 0$. This statement can be easily understood if we consider the case of $\gamma=1$, where for $0 \leq g<g_{c}$ the ground state has no magnetization in the $x$ direction: For $g=0$, the spins align with the magnetic field, while an infinitesimal spin interaction disorders the system and $M_{x}=0$. On the other hand, for $g \rightarrow \infty$ the states $\left|g_{1}\right\rangle=\frac{1}{\sqrt{2}}[|\rightarrow, \cdots, \rightarrow\rangle+|\leftarrow, \cdots, \leftarrow\rangle]$ and $\left|g_{2}\right\rangle=\frac{1}{\sqrt{2}}[|\rightarrow, \cdots, \rightarrow\rangle-|\leftarrow, \cdots, \leftarrow\rangle]$, with $|\rightarrow\rangle=\frac{1}{\sqrt{2}}[|\uparrow\rangle+|\downarrow\rangle]$ and $|\leftarrow\rangle=\frac{1}{\sqrt{2}}[|\uparrow\rangle-|\downarrow\rangle]$ become degenerate in the thermodynamic limit, and a ground state with $\left\langle\sigma_{x}^{i}\right\rangle \neq 0$ can be constructed from a linear combination.

Remarkably, this paramagnetic-to-ferromagnetic QPT does not exist in the isotropic limit $(\gamma=0)$. In this case, the Hamiltonian of Eq. (37) has a continuous $\mathfrak{u}(1)$ symmetry; that is, it is invariant under any $\hat{z}$ rotation of the form $\exp \left[i \theta \sum_{j} \sigma_{z}^{j}\right]$. Since the model is one-dimensional, this symmetry cannot be spontaneously broken, regardless of the magnitude of the coupling constants. Nevertheless, a simple calculation of the ground state energy indicates a divergence in its second derivative at the critical point $g_{c}=1 / 2$, thus, a second order non-broken symmetry QPT. For $g<g_{c}$ all the spins (in the ground state) are aligned with the external magnetic field, with total magnetization in the $\hat{z}$ direction $M_{z}=\sum_{j}\left\langle\sigma_{z}^{j}\right\rangle=-N$, and the quantum phase is gapped. For $g \geq g_{c}$, the total magnetization in the $\hat{z}$ direction is $M_{z} \geq-N$, the gap vanishes, and the 


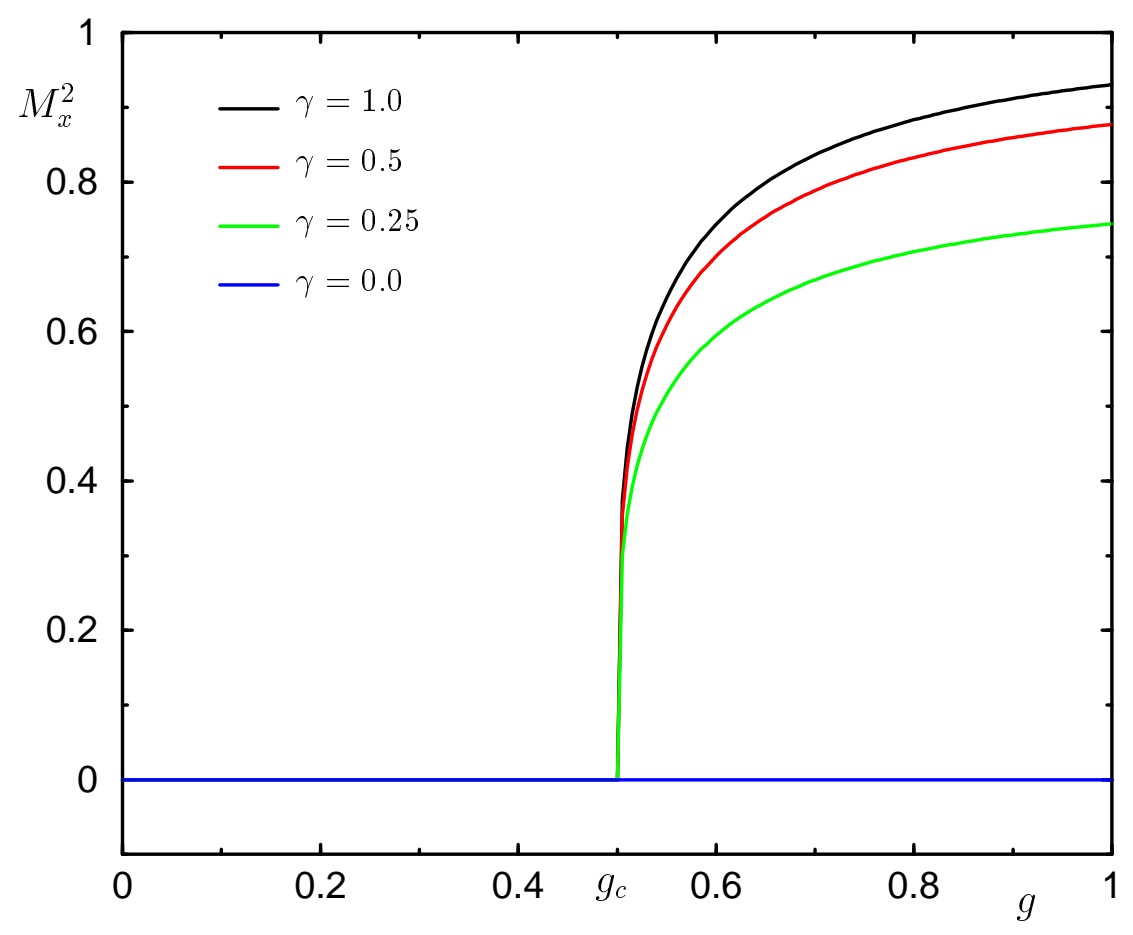

FIG. 8: Order parameter $M_{x}^{2}$ in the thermodynamic limit as a function of $g$ for different anisotropies $\gamma$. The critical point is at $g_{c}=1 / 2$.

quantum phase becomes critical (i.e., the spin-spin correlation functions decay with a power law), with an emergent $\mathfrak{u}(1)$ gauge symmetry [17]. Then, in terms of fermionic operators (Eq. (39), an insulator-metal (or superfluid) like second order QPT exists at $g_{c}$ for the isotropic case, with no symmetry order parameter. It is a Lifshitz transition.

\section{B. $\mathfrak{u}(N)$-purity in the BCS state, and critical behavior}

The $|\mathrm{BCS}\rangle$ state of Eq. (46) is a GCS of the algebra of observables $\mathfrak{h}=\mathfrak{s o}(2 N)$, spanned by an orthonormal Hermitian basis which is constructed by adjoining to the basis of $\mathfrak{u}(N)$ given in Eq. (20) the following set $\mathfrak{r}$ of number-non-conserving fermionic operators:

$$
\mathfrak{r}=\left\{\begin{array}{ll}
\left(c_{j}^{\dagger} c_{j^{\prime}}^{\dagger}+c_{j^{\prime}} c_{j}\right) & \text { with } 1 \leq j<j^{\prime} \leq N \\
i\left(c_{j}^{\dagger} c_{j^{\prime}}^{\dagger}-c_{j^{\prime}} c_{j}\right) & \text { with } 1 \leq j<j^{\prime} \leq N
\end{array} \quad, \quad \mathfrak{s o}(2 N)=\mathfrak{u}(N) \oplus \mathfrak{r} .\right.
$$

Then, the $|\mathrm{BCS}\rangle$ state is generalized unentangled with respect to the $\mathfrak{s o}(2 N)$ algebra and its purity $P_{\mathfrak{h}}$ (Eq. (7)) contains no information about the phase transition: $P_{\mathfrak{h}}=1 \forall g, \gamma$. Therefore, in 
order to characterize the QPT we need to look at the possible subalgebras of $\mathfrak{s o}(2 N)$. A natural choice is to restrict to operators which preserve the total fermion number that is, to consider the $\mathfrak{u}(N)$ algebra defined in Section $\amalg \mathrm{C}$ relative to which the $|\mathrm{BCS}\rangle$ state may become generalized entangled. (Note that as mentioned in Section $\amalg \mathrm{C}$ the $\mathfrak{u}(N)$ algebra can also be written in terms of the fermionic operators $\tilde{c}_{k}^{\dagger}$ and $\tilde{c}_{k}$, with $k$ belonging to the set $V$.)

In the $|\mathrm{BCS}\rangle$ state, $\left\langle\tilde{c}_{k}^{\dagger} \tilde{c}_{k^{\prime}}\right\rangle \neq 0$ only if $k=k^{\prime}$, thus using Eq. (21) the purity relative to $\mathfrak{h}=\mathfrak{u}(N)$ is:

$$
P_{\mathfrak{h}}(|\mathrm{BCS}\rangle)=\frac{4}{N} \sum_{k \in V}\left\langle\tilde{c}_{k}^{\dagger} \tilde{c}_{k}-1 / 2\right\rangle^{2}=\frac{4}{N} \sum_{k \in V}\left(v_{k}^{2}-1 / 2\right)^{2},
$$

where the coefficients $v_{k}$ can be obtained from Eqs. (43) and (44). In particular, for $g=0$ the spins are aligned with the magnetic field and the fully polarized $|\mathrm{BCS}\rangle_{g=0}=|\downarrow, \downarrow, \ldots, \downarrow\rangle$ state is generalized unentangled in this limit (a GCS of $\mathfrak{u}(N)$ with $P_{\mathfrak{h}}=1$ ). In the thermodynamic limit, the purity relative to the $\mathfrak{u}(N)$ algebra can be obtained by integrating Eq. (48):

$$
P_{\mathfrak{h}}(|\mathrm{BCS}\rangle)=\frac{2}{\pi} \int_{0}^{2 \pi}\left(v_{k}^{2}-1 / 2\right)^{2} d k
$$

leading to the following result:

$$
P_{\mathfrak{h}}(|\mathrm{BCS}\rangle)=\left\{\begin{array}{cc}
\frac{1}{1-\gamma^{2}}\left[1-\frac{\gamma^{2}}{\sqrt{1-4 g^{2}\left(1-\gamma^{2}\right)}}\right] & \text { if } g \leq 1 / 2 \\
\frac{1}{1+\gamma} & \text { if } g>1 / 2
\end{array} .\right.
$$

Although this function is continuous, its derivative is not and has a drastic change at $g=1 / 2$, where the QPT occurs. Moreover, $P_{\mathfrak{h}}$ is minimum for $g>1 / 2$ implying maximum entanglement at the transition point and in the ordered (ferromagnetic) phase. Remarkably, for $g>1 / 2$ and $N \rightarrow \infty$, where the ground state of the anisotropic XY model in a transverse magnetic field is two-fold degenerate, $P_{\mathfrak{h}}$ remains invariant for arbitrary linear combinations of the two degenerate states.

As defined, for large $g$ the purity $P_{\mathfrak{h}}$ approaches a constant value which depends on $\gamma$. It is convenient to remove such dependence in the ordered phase by introducing a new quantity $P_{\mathfrak{h}}^{\prime}=P_{\mathfrak{h}}-\frac{1}{1+\gamma}$ (shifted purity). We thus obtain

$$
P_{\mathfrak{h}}^{\prime}(|\mathrm{BCS}\rangle)=\left\{\begin{array}{cc}
\frac{\gamma}{1-\gamma^{2}}\left[1-\frac{\gamma}{\sqrt{1-4 g^{2}\left(1-\gamma^{2}\right)}}\right] & \text { if } g \leq 1 / 2 \\
0 & \text { if } g>1 / 2
\end{array} .\right.
$$

The new function $P_{\mathfrak{h}}^{\prime}$ behaves like a disorder parameter for the system, being zero in the ferromagnetic (ordered) phase and different from zero in the paramagnetic (ordered) one. The behavior 
of $P_{\mathfrak{h}}^{\prime}$ as a function of $g$ in the thermodynamic limit is depicted in Fig. 9 for different values of $\gamma$. In the special case of the Ising model in a transverse magnetic field $(\gamma=1)$, one has the simple behavior $P_{\mathfrak{h}}^{\prime}=1 / 2-2 g^{2}$ for $g \leq 1 / 2$ and $P_{\mathfrak{h}}^{\prime}=0$ if $g>1 / 2$.

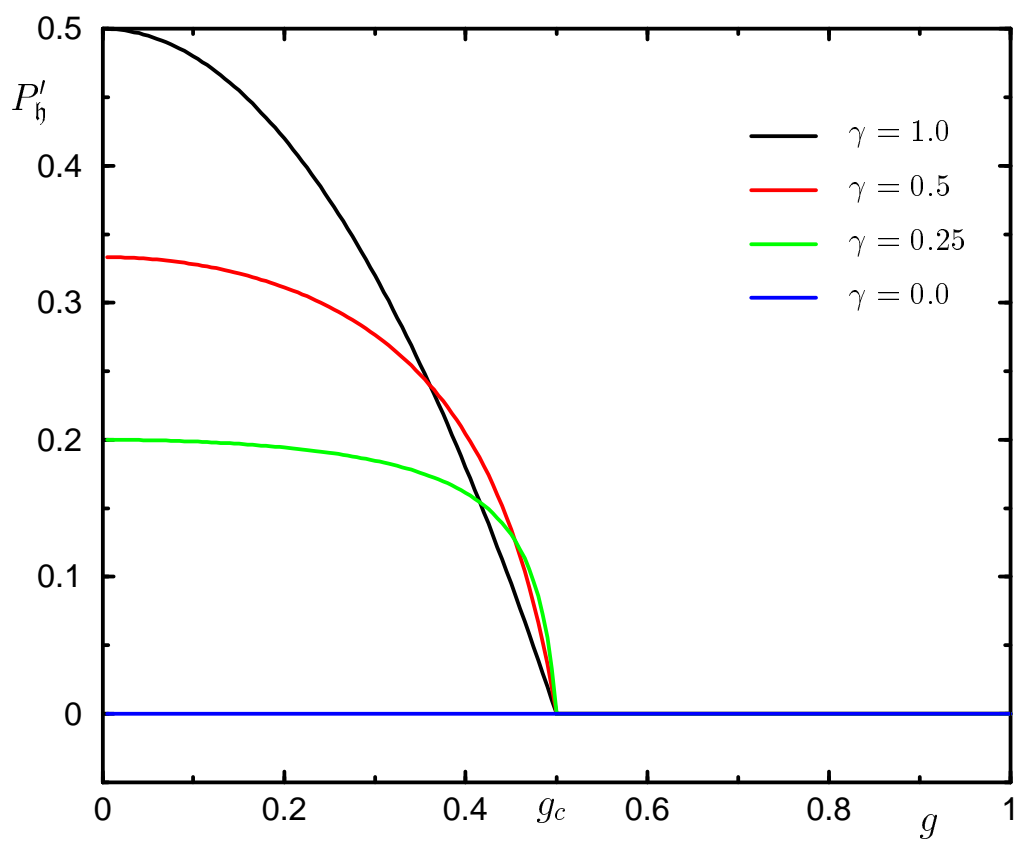

FIG. 9: Shifted purity $P_{\mathfrak{u}(N)}^{\prime}$ of the $\mid$ BCS $\rangle$ as a function of $g$ for different anisotropies $\gamma$, Eq. [51. $P_{\mathfrak{u}(N)}^{\prime}$ behaves like a disorder parameter for this model, sharply identifying the QPT at $g_{c}=1 / 2$.

The critical behavior of the system is characterized by a power-law divergence of the correlation length $\epsilon$, which is defined such that for $g<1 / 2, \lim _{|i-j| \rightarrow \infty}\left|\left\langle\sigma_{x}^{i} \sigma_{x}^{j}\right\rangle\right| \sim \exp \left(-\frac{|i-j|}{\epsilon}\right)$. Thus, $\epsilon \rightarrow \infty$ signals the emergence of long-range correlations in the ordered region $g>1 / 2$. Near the critical point $\left(g \rightarrow 1 / 2^{-}\right)$the correlation length behaves as $\epsilon \sim\left(g_{c}-g\right)^{-\nu}$, where $\nu$ is a critical exponent and the value $\nu=1$ corresponds to the Ising universality class. Let the parameter $\lambda_{2}=e^{-1 / \epsilon}$. The fact that the purity contains information about the critical properties of the model follows from the possibility of expressing $P_{\mathfrak{h}}^{\prime}$ for $g<1 / 2$ as a function of the correlation length,

$$
P_{\mathfrak{h}}^{\prime}(|\mathrm{BCS}\rangle)=\frac{\gamma}{1-\gamma^{2}}\left[1+\frac{\gamma}{2 g \lambda_{2}(1-\gamma)-1}\right]
$$

where a known relation between $g, \gamma$, and $\lambda_{2}$ has been exploited [4]. Performing a Taylor expansion of Eq. (52) in the region $g \rightarrow 1 / 2^{-}$, we obtain $P_{\mathfrak{h}}^{\prime} \sim 2\left(g_{c}-g\right)^{\nu} / \gamma$ with $\nu=1$ and $\gamma>0$ (Fig. 10). Thus, the name disorder parameter for $P_{\mathfrak{h}}^{\prime}$ is consistent. 


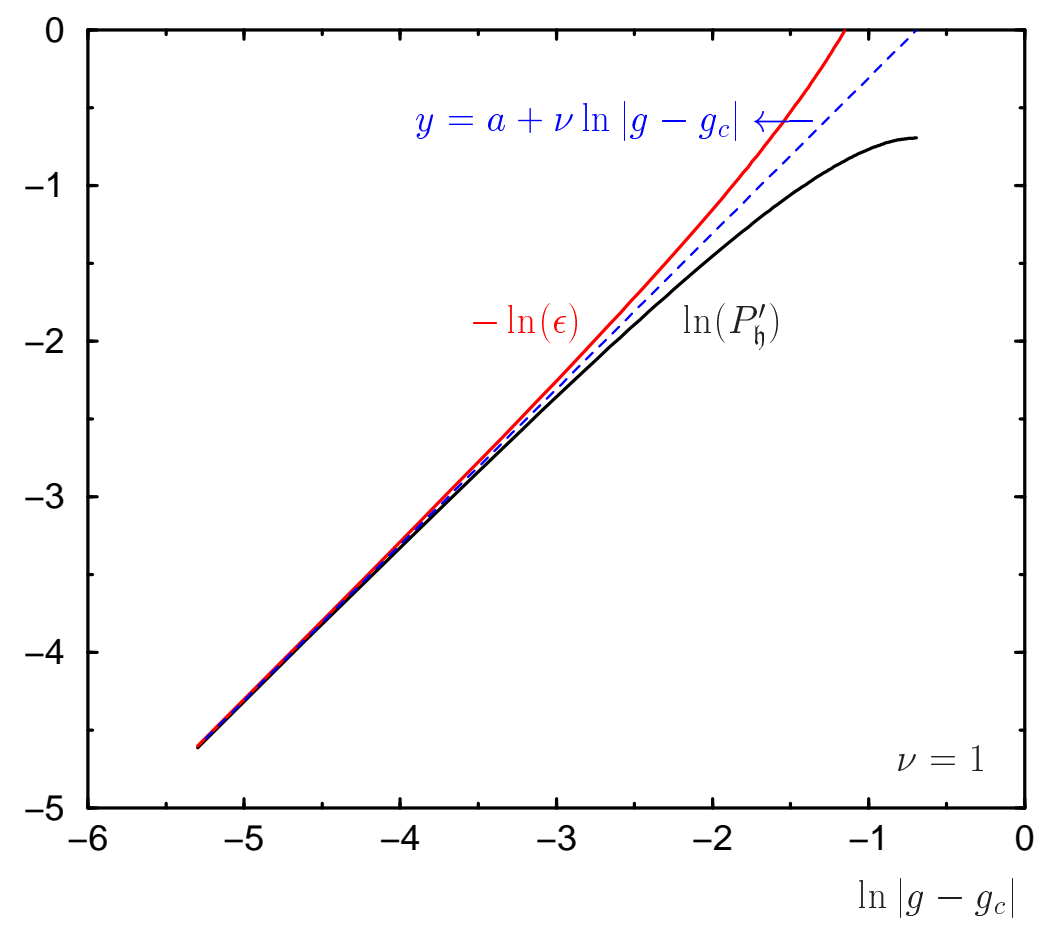

FIG. 10: Scaling properties of the disorder parameter for anisotropy $\gamma=1$. The exponent $\nu=1$ belongs to the Ising universality class.

Some physical insight in the meaning of the ground-state purity may be gained by noting that Eq. (48) can be written in terms of the fluctuations of the total fermion operator $\hat{N}$

$$
P_{\mathfrak{h}}(|\mathrm{BCS}\rangle)=1-\frac{2}{N}\left(\left\langle\hat{N}^{2}\right\rangle-\langle\hat{N}\rangle^{2}\right) .
$$

where the $|\mathrm{BCS}\rangle$-property $\left\langle\tilde{c}_{k}^{\dagger} \tilde{c}_{k^{\prime}}\right\rangle=\delta_{k, k^{\prime}} v_{k}^{2}$ has been used. In general, the purity relative to a given algebra can be written in terms of fluctuations of observables [15]. Since fluctuations of observables are at the root of QPTs it is not surprising that this quantity succeeds at identifying the critical point. Interestingly, by recalling that $P_{\mathfrak{s o}(2 N)}(|\mathrm{BCS}\rangle)=1$, the $\mathfrak{u}(N)$-purity can also be formally expressed as

$$
P_{\mathfrak{u}(N)}(|\mathrm{BCS}\rangle)=1-\sum_{A_{\alpha} \in \mathfrak{r}}\left\langle A_{\alpha}\right\rangle^{2},
$$

where the sum only extends to the non-number-conserving $\mathfrak{s o}(2 N)$-generators belonging to the set $\mathfrak{r}$ specified in Eq. (47). Thus, the purity is entirely contributed by expectations of operators connecting different $\mathfrak{u}(N)$-irreps, the net effect of correlating representations with a different particle number resulting in the fluctuation of a single operator, given by $\hat{N}=\sum_{k} \tilde{c}_{k}^{\dagger} \tilde{c}_{k}$. In Fig. 11 
we show the probability $\Omega(n)$ of having $n$ fermions in a chain of $N=400$ sites for $\gamma=1$. We observe that for $g>1 / 2$ the fluctuations remain almost constant, and so does the purity.

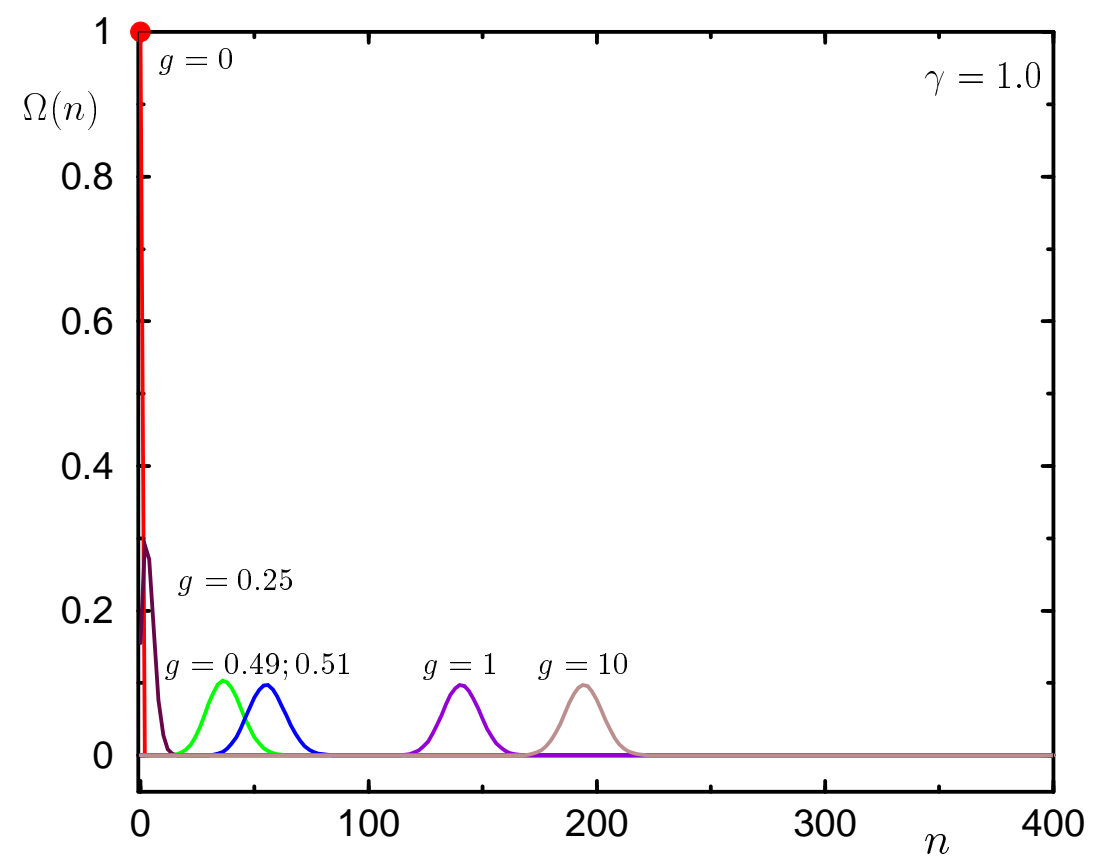

FIG. 11: Distribution of the fermion number in the $|\mathrm{BCS}\rangle$ state for a chain of $N=400$ sites and anisotropy $\gamma=1$

Again, the isotropic case $\left(\gamma=0\right.$ ) is particular in the sense that $P_{\mathfrak{h}}=1$ (or $P_{\mathfrak{h}}^{\prime}=0$, see Fig. 9), without identifying the corresponding metal-insulator QPT. The reason is that in this limit, the Hamiltonian of Eq. (39) contains only fermionic operators that preserve the number of particles (i.e., $H \in \mathfrak{u}(N)$ ), and the ground state of the system is always a GCS of the $\mathfrak{u}(N)$ algebra. Therefore, in order to obtain information about this QPT, one should look into algebras other than $\mathfrak{u}(N)$, relative to which the ground state is generalized entangled. For example, in Sec. VID we study the purity relative to the local algebra of observables and in Fig. 13 we show that it succesfully identifies the QPT in the isotropic case, being maximum for $g \leq g_{c}$ (thus implying generalized unentanglement).

\section{Comparison with concurrence}

As mentioned, the critical behavior of the XY model in a transverse field has also been investigated by looking at various quantities related to the concurrence, which is intrinsically a measure 
of bipartite entanglement. For a generic mixed state $\rho$ of two qubits, the latter is calculated as [14]

$$
C(\rho)=\max \left\{\lambda_{1}-\lambda_{2}-\lambda_{3}-\lambda_{4}, 0\right\}
$$

where $\lambda_{1} \geq \ldots \geq \lambda_{4}$ are the square roots of the eigenvalues of the matrix $R=\rho \tilde{\rho}$ and $\tilde{\rho}=\sigma_{y} \otimes$ $\sigma_{y} \rho^{*} \sigma_{y} \otimes \sigma_{y}$. The concurrence for the reduced density operator $\rho_{\ell, m}$ of two nearest-neighbor qubits $(|\ell-m|=1)$ and next-nearest-neighbor $(|\ell-m|=2)$ qubits on a lattice has been investigated in detail in Ref. [8]. Since, thanks to translational invariance, $\rho_{\ell, m}$ depends on the qubit indexes only via their distance, we will use the notation $C(1), C(2)$ for the resulting quantities as in [8]. While the results reported in the above work nicely agree with the scaling behavior expected for this model, the emerging picture based on concurrence cannot be regarded as fully satisfactory. As stressed in [8], the entanglement as quantified by the nearest-neighbor concurrence is not directly an indicator of the QPT in this model, showing maximum entanglement at a point which is not related to the QPT. However, the derivative $\partial C(1) / \partial g$ of the concurrence with respect to the spinspin coupling parameter can be seen to diverge logarithmically at the critical point for $\gamma>0$, and with a power law for the isotropic case [Fig. [12] ], identifying the critical point in this model. Such a divergence is not found when analyzing, at the isotropic point, other QPTs in models of interest, like the one-dimensional anisotropic Heisenberg chain (see, for instance, [51]). Therefore, it suggests that the identification of a critical point using concurrence could be a hard task in general.

\section{Purity of the BCS state relative to the local algebra}

Finally, we have also investigated the behavior of the purity of the $|\mathrm{BCS}\rangle$ state relative to the algebra of local observables $\mathfrak{h}=\bigoplus_{i=1}^{N} \mathfrak{s u}(2)_{i}$. Using Eq. (16), this is physically related to the total magnetization $M_{z}^{2}$ along $z$. The resulting behavior is plotted in Fig. 13 as a function of $g$ and $\gamma$. As explained in Section $1 \mathrm{IIB}$, this is a measure of the usual notion of entanglement in the $N$-spin-1/2s system. In particular, the $|\mathrm{BCS}\rangle$ state is unentangled for $g \rightarrow 0$ (where $|\mathrm{BCS}\rangle \sim$ $\left.\left|-\frac{1}{2}\right\rangle_{1} \otimes \cdots \otimes\left|-\frac{1}{2}\right\rangle_{N}\right)$, thus $P_{\mathfrak{h}} \rightarrow 1$ in this limit. Moreover, for $g \rightarrow \infty$ we have $|\mathrm{BCS}\rangle \sim\left|\mathrm{GHZ}_{\frac{1}{2}}^{N}\right\rangle$ (up to local rotations), thus $|\mathrm{BCS}\rangle$ becomes maximally entangled, and $P_{\mathfrak{h}} \rightarrow 0$.

Compared to the purity relative to the $\mathfrak{u}(N)$ algebra, the purity relative to $\mathfrak{h}=\bigoplus_{i=1}^{N} \mathfrak{s u}(2)_{i}$ is not as good an indicator of the phase transition when $\gamma>0$, in the sense that it does not present any drastic change in its behavior. However, its derivative with respect to the spin-spin coupling 


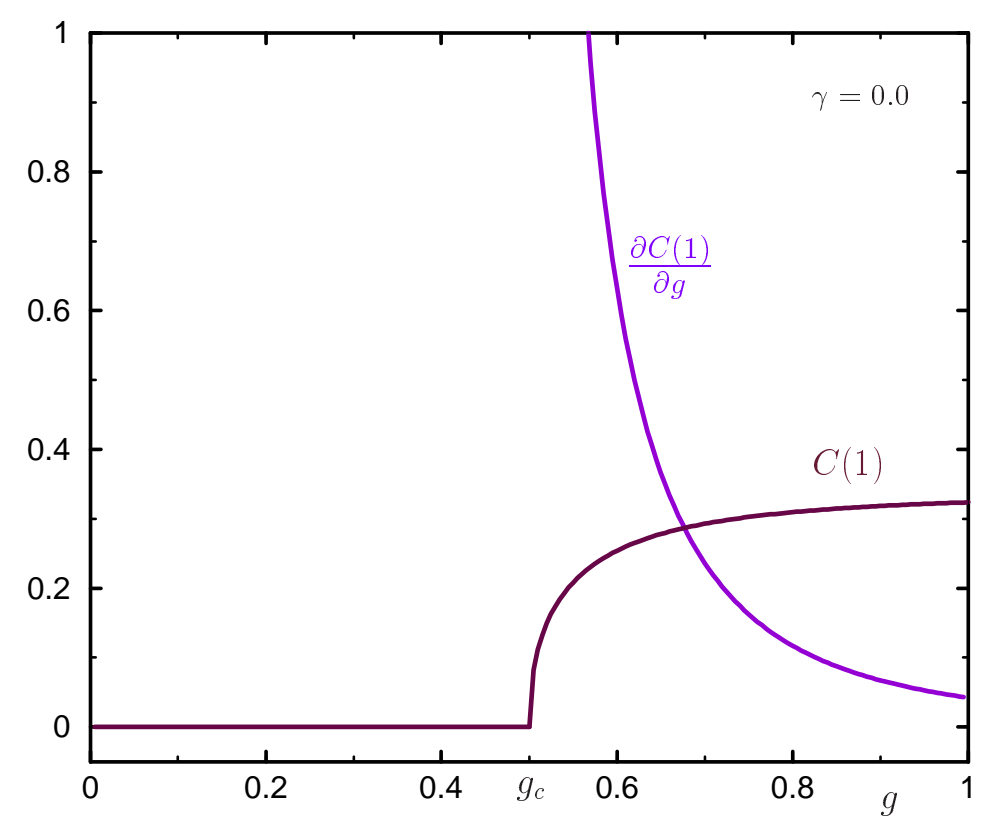

FIG. 12: Nearest-neighbor concurrence and its derivative for the $|\mathrm{BCS}\rangle$ state as a function of $g$ in the isotropic $\mathrm{XY}$ model, $\gamma=0$. Both curves correspond to the exact solution in the thermodynamic limit. The value of $\partial C(1) / \partial g$ below $g_{c}$ is also zero as $C(1)$ (not shown).

parameter diverges at the critical point in this model [Fig. 14]. Only in the isotropic case $(\gamma=0)$ the purity relative to the local algebra presents a drastic change at the critical point (see Fig. 13). In this case, the operator $M_{z}=\frac{1}{N}\left\langle\sum_{j} \sigma_{z}^{j}\right\rangle$ for $g \rightarrow g_{c}^{+}$scales as

$$
M_{z}+1 \sim\left(g-g_{c}\right)^{\chi}
$$

with the exponent being $\chi=1 / 2$. On the other hand, this exponent can also be obtained from the purity relative to the local algebra, in the same limit:

$$
1-P_{\mathfrak{h}} \sim\left(g-g_{c}\right)^{\chi} .
$$

Therefore, this measure of entanglement is also a good indicator of the QPT for the isotropic case.

\section{CONCLUSIONS}

In this paper, we have explored the usefulness of generalized entanglement (GE) for characterizing the broken (and one example of non-broken) symmetry quantum phase transitions (QPTs) 


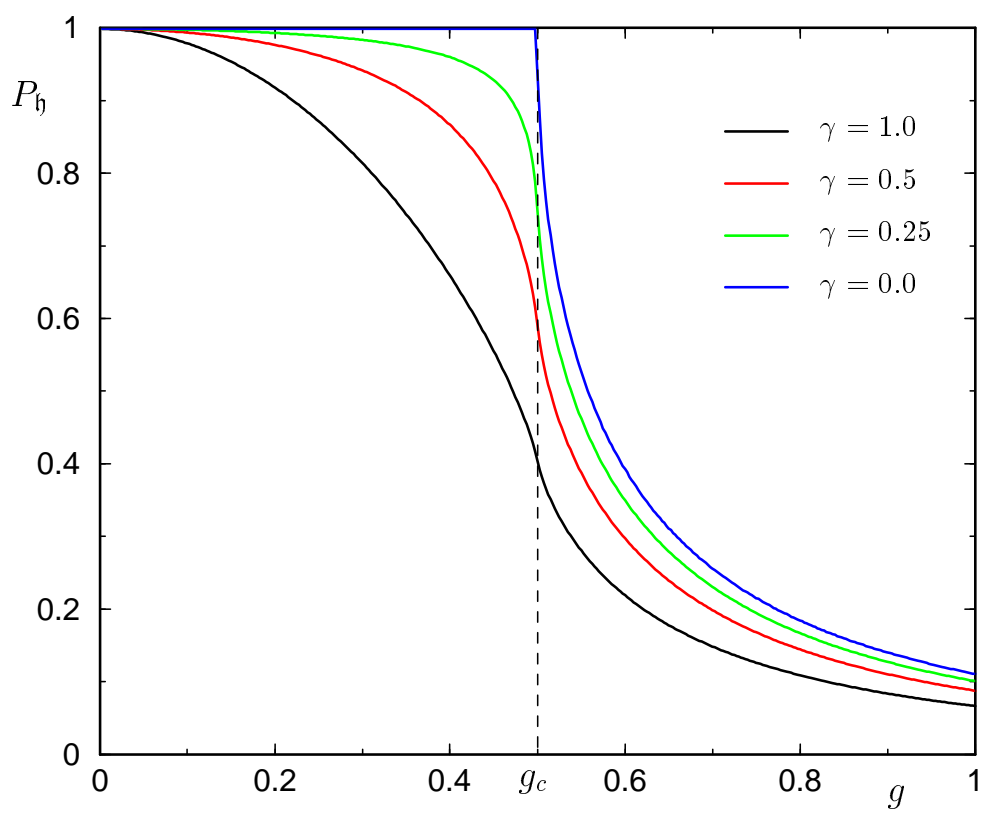

FIG. 13: Purity of the $|\mathrm{BCS}\rangle$ state relative to the local algebra $\bigoplus_{i=1}^{N} \mathfrak{s u}(2)_{i}$, as a function of $g$ for different anisotropies $\gamma\left(g_{c}=1 / 2\right)$. The number of sites $N=400$ as in Fig. 11

present in different lattice systems. As we focused on situations where the physically relevant observables form a Lie algebra, a natural GE measure provided by the relative purity of a state relative to the algebra has been used to identify and characterize these transitions.

In Sections purity can be useful for different spin systems, by encompassing the usual notion of entanglement if the family of all local observables is distinguished. In addition, the possibility to directly apply the GE notion to arbitrary quantum systems, including indistinguishable particles, was explicitly shown in Section $\amalg \mathrm{C}$, using fermionic systems as a relevant case study. Depending on the subset of observables chosen, the $\mathfrak{h}$-purity contains information about different $n$-body correlations present in the quantum state, allowing for a more general and complete characterization of entanglement. Finally, in Sections $\nabla$ and $\nabla]$ we showed that the $\mathfrak{h}$-purity successfully distinguishes between the different phases present in two lattice systems, where the critical points are characterized by a broken symmetry (or non-broken symmetry in the case of the isotropic XY model in an external magnetic field) and the usual notion of entanglement cannot be straightforwardly applied. As also discussed in Section IV the most critical step is to determine which subset of 


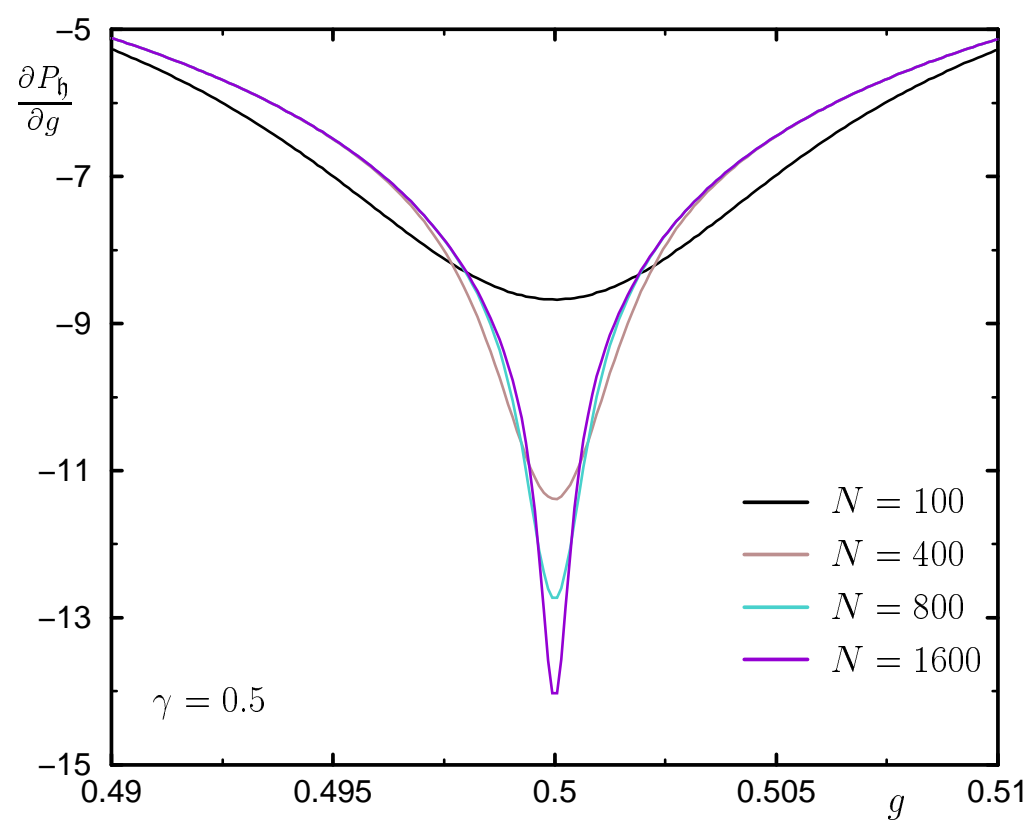

FIG. 14: Derivative of the purity of the $|\mathrm{BCS}\rangle$ state relative to the local algebra as a function of $g$ for $\gamma=0.5$ and different lattice sizes.

observables may be relevant in each case, since the $\mathfrak{h}$-purity must contain information about the quantum correlations that play a dominant role in the QPT. In particular, the ground state of the two models we considered can be exactly calculated and the relevant quantum correlations in the different phases are well understood, thus choosing this subset of observables becomes relatively easy.

Applying these concepts to a more general case, where the ground state of the system cannot be exactly computed, can be done in principle by following the same strategy. However, determining in a systematic way the minimal subset of observables $\mathfrak{h}$ whose purity is able to signal and characterize the QPT, thereby providing the relevant correlations, requires an elaborate analysis. Even more interesting, perhaps, is the open question of finding the minimal number of GE measures, possibly including measures of GE relative to different observable sets, needed to unambiguously characterize the universality class of a transition, obtaining all of its critical exponents. Finally, a fascinating direction for further investigation is to explore the significance of the GE notion within topological quantum-information settings [52] and to understand what generalizations might be 
needed to handle topological QPTs.

\section{Acknowledgments}

It is a pleasure to thank James Gubernatis, Leonid Gurvits, Juan Pablo Paz, and Wojciech Zurek for discussions. We acknowledge support from the US DOE through Contract No. W-7405-ENG36. H. B. and L. V. gratefully acknowledge financial support from the Los Alamos Office of the Director.

\section{APPENDIX A: SEPARABILITY, GENERALIZED UNENTANGLEMENT, AND LOCAL PURI-} TIES

Given a quantum system $\mathcal{S}$ whose states $|\psi\rangle$ belong to a Hilbert space $\mathcal{H}$ of dimension $\operatorname{dim}(\mathcal{H})=d$, the purity relative to the (real) Lie algebra of all traceless observables $\mathfrak{h}=\mathfrak{s u}(d)$ spanned by an orthogonal, commonly normalized Hermitian basis $\left\{A_{1} \cdots A_{L}\right\}, L=d^{2}-1$, is, according to Eq. (7), given by:

$$
P_{\mathfrak{h}}(|\psi\rangle)=\mathrm{K} \sum_{\alpha=1}^{L}\left\langle A_{\alpha}\right\rangle^{2} .
$$

The normalization factor $\mathrm{K}$ depends on $d$ and is determined so that the maximum purity value is 1 . If $\operatorname{Tr}\left(A_{\alpha} A_{\beta}\right)=\delta_{\alpha, \beta}$ (as for the standard spin-1 Gell-Mann matrices), then $\mathrm{K}=d /(d-1)$, whereas in the case $\operatorname{Tr}\left(A_{\alpha} A_{\beta}\right)=d \delta_{\alpha, \beta}$ (as for ordinary spin-1/2 Pauli matrices), $\mathrm{K}=1 /(d-1)$. Recall that any quantum state $|\psi\rangle \in \mathcal{H}$ can be obtained by applying a group operator $U$ to a reference state $\mid$ ref $\rangle$ (a highest or lowest weight state of $\mathfrak{s u}(d)$ ); that is

$$
|\psi\rangle=U|\mathrm{ref}\rangle
$$

with $U=e^{i \sum_{\alpha} t_{\alpha} A_{\alpha}}$, and $t_{\alpha}$ real numbers. Therefore, any quantum state $|\psi\rangle$ is a GCS of $\mathfrak{s u}(d)$, thus generalized unentangled relative to the algebra of all observables: $P_{\mathfrak{h}}(|\psi\rangle)=1$ for all $|\psi\rangle$.

Let now assume that $\mathcal{S}$ is composed of $N$ distinguishable susbsytems, corresponding to a factorization $\mathcal{H}=\bigotimes_{j=1}^{N} \mathcal{H}_{j}$, with $\operatorname{dim}\left(\mathcal{H}_{j}\right)=d_{j}, d=\prod_{j} d_{j}$. Then the set of all local observables on $\mathcal{S}$ becomes $\mathfrak{h}=\mathfrak{h}_{l o c}=\bigoplus_{j} \mathfrak{s u}\left(d_{j}\right)$. An orthonormal basis which is suitable for calculating the local purity $P_{\mathfrak{h}}$ may be obtained by considering a collection of orthonormal bases $\left\{A_{\alpha_{1}}^{j} \cdots A_{\alpha_{L_{j}}}^{j}\right\}$, 
$L_{j}=d_{j}^{2}-1$, each acting on the $j$ th subsystem that is,

$$
A_{\alpha_{j}}^{j}=\overbrace{\mathbb{1}^{1} \otimes \mathbb{1}^{2} \otimes \cdots \otimes \underbrace{A_{\alpha_{j}}}_{j^{\text {th }} \text { factor }} \otimes \cdots \otimes \mathbb{1}^{N}}^{N \text { factors }},
$$

where $\mathbb{1}^{j}=\mathbb{1} / \sqrt{d_{j}}$. Then for any pure state $|\psi\rangle \in \mathcal{H}$ one may write

$$
P_{\mathfrak{h}}(|\psi\rangle)=\mathrm{K}^{\prime} \sum_{j=1}^{N}\left[\sum_{\alpha_{j}=1}^{L_{j}}\left\langle A_{\alpha_{j}}^{j}\right\rangle^{2}\right] .
$$

By letting $\mathfrak{h}_{j}=\operatorname{span}\left\{A_{\alpha_{j}}\right\}$ be the Lie algebra of traceless Hermitian operators acting on $\mathcal{H}_{j}$ alone, the above equation also is naturally rewritten as

$$
P_{\mathfrak{h}}(|\psi\rangle)=\mathrm{K}^{\prime} \sum_{j=1}^{N} \frac{1}{\mathrm{~K}_{j}} P_{\mathfrak{h}_{j}}(|\psi\rangle), \quad \mathrm{K}_{j}=\frac{d_{j}}{d_{j}-1} .
$$

The $\mathfrak{h}_{j}$-purity $P_{\mathfrak{h}_{j}}$ may be simply related to the conventional subsystem purity. Let $\rho_{j}=$ $\operatorname{Tr}_{i \neq j}(\{|\psi\rangle\langle\psi|\})$ be the reduced density operator describing the state of the $j$ th subsystem. Because the latter can be represented as

$$
\rho_{j}=\frac{1}{d_{j}}+\sum_{\alpha_{j}=1}^{L_{j}}\left\langle A_{\alpha_{j}}\right\rangle A_{\alpha_{j}}=\sum_{\alpha_{j}=1}^{L_{j}}\left\langle A_{\alpha_{j}}^{j}\right\rangle A_{\alpha_{j}},
$$

one can also equivalently express Eq. (A4) as

$$
P_{\mathfrak{h}}(|\psi\rangle)=\mathrm{K}^{\prime} \sum_{j=1}^{N}\left[\operatorname{Tr} \rho_{j}^{2}-\frac{1}{d_{j}}\right],
$$

that is, $P_{\mathfrak{h}_{j}}(|\psi\rangle)=\left(d_{j} \operatorname{Tr} \rho_{j}^{2}-1\right) /\left(d_{j}-1\right)$. Clearly, the maximum value of either Eqs. A5 or A7] will be attained when, and only when, each of the conventional purities $\operatorname{Tr} \rho_{j}^{2}=1 \leftrightarrow P_{\mathfrak{h}_{j}}=1$ for all $j$, which allows determining the $\mathrm{K}^{\prime}$-normalization factor as

$$
\mathrm{K}^{\prime}=\frac{1}{\sum_{j} \frac{1}{\mathrm{~K}_{j}}}=\frac{1}{N-\sum_{j} \frac{1}{d_{j}}}=\frac{1}{N\left(1-\frac{1}{N} \sum_{j} \frac{1}{d_{j}}\right)} .
$$

Accordingly,

$$
P_{\mathfrak{h}_{l o c}}(|\psi\rangle)=\max =1 \leftrightarrow|\psi\rangle=\left|\phi_{1}\right\rangle \otimes \cdots \otimes\left|\phi_{N}\right\rangle
$$

and the equivalence with the standard notions of separability and entanglement are recovered. Note that for the case of $N$ qubits considered in Section IIIB, the above value simplifies to $\mathrm{K}^{\prime}=$ $2 / N$ which in turn gives the purity expression of Eq. (16) once the standard unnormalized Pauli matrices are used $\left(A_{\alpha_{j}}^{j}=\sigma_{\alpha_{j}}^{j} / \sqrt{2}\right.$, thus removing the overall factor 2 ). 


\section{APPENDIX B: CLUSTER AND VALENCE BOND SOLID STATES ARE MAXIMALLY EN-}

\section{TANGLED}

In Ref. [38], Briegel and Raussendorf introduced the so-called cluster states for a system of $N$ qubits in $D$ space dimensions which, in the computational basis, are expressed as

$$
|\Phi\rangle_{C}=\frac{1}{2^{N / 2}} \bigotimes_{j \in C}\left(|\uparrow\rangle_{j} \prod_{\gamma \in \Gamma} \sigma_{z}^{(j+\gamma)}+|\downarrow\rangle_{j}\right),
$$

where $C$ defines the cluster $\left(C \subset \mathbb{Z}^{D}\right)$ and $\gamma$ denotes some nearest neighbor qubits in the cluster: $\Gamma=\{1\}$ for $D=1, \Gamma=\{(1,0),(0,1)\}$ for $D=2, \Gamma=\{(1,0,0),(0,1,0),(0,0,1)\}$ for $D=3$, etc. We consider $\sigma_{z}^{(j+\gamma)} \equiv 1$ when $j+\gamma$ is not in $C$.

The usual notion of entanglement, as applied to a cluster state, is recovered when the $\mathfrak{h}$-purity is calculated relative to the local algebra $\mathfrak{h}=\bigoplus_{j \in C} \mathfrak{s u}(2)_{j}$ (see Appendix $\mathrm{\AA}$. For this purpose, we first calculate the expectation values $\left\langle\sigma_{\alpha}^{j}\right\rangle_{C}$, with $\alpha=x, y, z$. One can immediately realize that $\left\langle\sigma_{y}^{j}\right\rangle_{C}=0, \forall j$, since $\sigma_{y}^{j}$ is an Hermitian operator (i.e., $\left\langle\sigma_{y}^{j}\right\rangle \in \mathbb{R}$ ) that acting on the $j$-th qubit's state (in the natural basis) introduces a phase factor $\pm i$, and the coefficients of Eq. (B1) are all real. Moreover, $\left\langle\sigma_{z}^{j}\right\rangle_{C}=0, \forall j$, since the weight of every state of the natural basis is the same in Eq. (B1). In other words, we have a linear combination of basis states where each single qubit has the same probability of pointing up or down. Finally, one can also prove that $\left\langle\sigma_{x}^{j}\right\rangle_{C}=0, \forall j$. This can be done by using the eigenvalue equations $K_{j}|\Phi\rangle_{C}= \pm|\Phi\rangle_{C}$, for the family of operators $K_{j}=\sigma_{x}^{j} \prod_{\gamma \in \bar{\Gamma}} \sigma_{z}^{(j+\gamma)}$, where $\bar{\Gamma}=\Gamma \bigcup-\Gamma$ denotes the set of all nearest-neighbor qubits to the $j$-th qubit. Therefore, $\left\langle\sigma_{x}^{j}\right\rangle_{C}= \pm\left\langle\sigma_{x}^{j} K_{j}\right\rangle_{C}= \pm\left\langle\prod_{\gamma \in \bar{\Gamma}} \sigma_{z}^{(j+\gamma)}\right\rangle_{C}$. Again, since Eq. (B1) is a combination of all the states of the computational basis with the same probability, we obtain $\left\langle\sigma_{x}^{j}\right\rangle_{C}=0$. In this way, the $\mathfrak{h}$-purity (Eq. (16) is $P_{\mathfrak{h}}=0$, and the cluster states are maximally entangled relative to the local set $\mathfrak{h}=\bigoplus_{j \in C} \mathfrak{s u}(2)_{j}$.

Another important class of spin states is the one defined by the so-called Valence Bond Solid (VBS) states. These states have been introduced in the context of Heisenberg-like magnets, and have been recently revisited in the context of quantum computation [50]. Their general form is

$$
|\Phi\rangle_{\mathrm{VBS}}=\prod_{\langle i, j\rangle}\left(a_{i}^{\dagger} b_{j}^{\dagger}-b_{i}^{\dagger} a_{j}^{\dagger}\right)^{M}|0\rangle,
$$

where $\langle i, j\rangle$ represent nearest-neighbor bonds of a $D$-dimensional lattice of coordination z, $a_{j}^{\dagger}$ and $b_{j}^{\dagger}$ are Schwinger-Wigner boson (creation) operators on site $j$ whose relation to $\mathfrak{s u}(2) \operatorname{spin}-S$ 
generators is

$$
S_{x}^{j}=\frac{1}{2}\left(a_{j}^{\dagger} b_{j}+b_{j}^{\dagger} a_{j}\right), \quad S_{y}^{j}=\frac{1}{2 i}\left(a_{j}^{\dagger} b_{j}-b_{j}^{\dagger} a_{j}\right), \quad S_{z}^{j}=\frac{1}{2}\left(a_{j}^{\dagger} a_{j}-b_{j}^{\dagger} b_{j}\right),
$$

with the constraint $a_{j}^{\dagger} a_{j}+b_{j}^{\dagger} b_{j}=2 S$, and $M=2 S /$ z. $M$ being an integer makes the possible values of $S$ to depend upon the connectivity of the lattice, which is defined by z.

We start by showing that the bond operators $a_{i}^{\dagger} b_{j}^{\dagger}-b_{i}^{\dagger} a_{j}^{\dagger}$ are invariant under global spin rotations. The Schwinger-Wigner boson operators transform as vectors for $\mathfrak{s u}(2)$ rotations

$$
\left(\begin{array}{c}
a_{j}^{\dagger} \\
b_{j}^{\dagger}
\end{array}\right) \rightarrow U_{j}\left(\begin{array}{c}
a_{j}^{\dagger} \\
b_{j}^{\dagger}
\end{array}\right) U_{j}^{\dagger}=\left(\begin{array}{cc}
\cos \frac{\theta_{2}}{2} e^{i\left(\theta_{3}+\theta_{1}\right) / 2} & \sin \frac{\theta_{2}}{2} e^{i\left(\theta_{3}-\theta_{1}\right) / 2} \\
-\sin \frac{\theta_{2}}{2} e^{-i\left(\theta_{3}-\theta_{1}\right) / 2} & \cos \frac{\theta_{2}}{2} e^{-i\left(\theta_{3}+\theta_{1}\right) / 2}
\end{array}\right)\left(\begin{array}{c}
a_{j}^{\dagger} \\
b_{j}^{\dagger}
\end{array}\right)
$$

under an arbitrary spin rotation on lattice site $j$, defined by

$$
U_{j}=e^{i \theta_{1} S_{z}^{j}} e^{i \theta_{2} S_{y}^{j}} e^{i \theta_{3} S_{z}^{j}}, \quad U_{j} U_{j}^{\dagger}=U_{j}^{\dagger} U_{j}=\mathbb{1} .
$$

Then, we can use this result to prove that

$$
U_{j} U_{i}\left(a_{i}^{\dagger} b_{j}^{\dagger}-b_{i}^{\dagger} a_{j}^{\dagger}\right) U_{i}^{\dagger} U_{j}^{\dagger}=a_{i}^{\dagger} b_{j}^{\dagger}-b_{i}^{\dagger} a_{j}^{\dagger},
$$

implying, for $U^{\dagger}=\prod_{j} U_{j}^{\dagger}$,

$$
U^{\dagger}|\Phi\rangle_{\mathrm{VBS}}=|\Phi\rangle_{\mathrm{VBS}}
$$

Therefore, $|\Phi\rangle_{\mathrm{VBS}}$ belongs to the singlet irrep of the total spin $J_{\alpha}=\sum_{j} S_{\alpha}^{j}$ (i.e., $\left\langle J_{\alpha}\right\rangle_{\mathrm{VBS}}=0$ ).

We want to show now that $\left\langle S_{\alpha}^{j}\right\rangle_{\mathrm{VBS}}=0, \forall j$. We first observe that $\left\langle S_{z}^{j}\right\rangle_{\mathrm{VBS}}=0, \forall j$, because the transformation that maps $a_{j}^{\dagger} \mapsto b_{j}^{\dagger}$ and $b_{j}^{\dagger} \mapsto-a_{j}^{\dagger}$ (i.e., a global spin rotation about the $y$-axis, setting $\theta_{1}=\theta_{3}=0$ and $\theta_{2}=\pi$ in Eq. (B) implies $\left\langle a_{j}^{\dagger} a_{j}\right\rangle_{\mathrm{VBS}}=\left\langle b_{j}^{\dagger} b_{j}\right\rangle_{\mathrm{VBS}}$. Then, from the invariance under global spin rotations and the singlet nature of $|\Phi\rangle_{\text {VBS }}$, we obtain $\left\langle S_{x}^{j}\right\rangle_{\text {VBS }}=0=$ $\left\langle S_{y}^{j}\right\rangle_{\mathrm{VBS}}, \forall j$. In other words, the purity relative to the algebra $\mathfrak{h}=\bigoplus_{j} \mathfrak{s u}(2)_{j}$ vanishes, meaning that $|\Phi\rangle_{\text {VBS }}$ is maximally generalized entangled relative to this algebra.

However, in order to make contact with the standard notion of entanglement (Appendix $\mathrm{A}$ ) we need to address the GE relative to the algebra $\mathfrak{h}=\bigoplus_{j} \mathfrak{s u}(2 S+1)_{j}$, that is, relative to the set of all local observables. For simplicity, we only discuss the $1 D$ case for $S=1$ (i.e., $M=1$ in Eq. (B2) but the reader could use the same techniques to obtain results in higher $D$ dimensions and spin magnitude $S$.

The algebra $\mathfrak{h}=\bigoplus_{j} \mathfrak{s u}(3)_{j}=\left\{\mathcal{S}_{\mu \nu}^{j}\right\}$,

$$
\left[\mathcal{S}_{\mu \mu^{\prime}}^{j}, \mathcal{S}_{\nu \nu^{\prime}}^{j^{\prime}}\right]=\delta_{j j^{\prime}}\left(\delta_{\mu^{\prime} \nu} \mathcal{S}_{\mu \nu^{\prime}}^{j}-\delta_{\mu \nu^{\prime}} \mathcal{S}_{\nu \mu^{\prime}}^{j}\right)
$$


can be written in terms of the $\mathfrak{s u}(2)$ generators as [17]

$$
\begin{aligned}
& \mathcal{S}_{00}^{j}=\frac{2}{3}-\left(S_{z}^{j}\right)^{2}, \mathcal{S}_{11}^{j}=\frac{S_{z}^{j}\left(S_{z}^{j}+1\right)}{2}-\frac{1}{3}, \\
& \mathcal{S}_{10}^{j}=\frac{1}{2 \sqrt{2}}\left[S_{+}^{j}+\left\{S_{+}^{j}, S_{z}^{j}\right\}\right] \\
& \mathcal{S}_{01}^{j}=\frac{1}{2 \sqrt{2}}\left[S_{-}^{j}+\left\{S_{-}^{j}, S_{z}^{j}\right\}\right] \\
& \mathcal{S}_{20}^{j}=\frac{1}{2 \sqrt{2}}\left[S_{-}^{j}-\left\{S_{-}^{j}, S_{z}^{j}\right\}\right] \\
& \mathcal{S}_{02}^{j}=\frac{1}{2 \sqrt{2}}\left[S_{+}^{j}-\left\{S_{+}^{j}, S_{z}^{j}\right\}\right] \\
& \mathcal{S}_{12}^{j}=\frac{i}{2}\left\{S_{x}^{j}, S_{y}^{j}\right\}+\left(S_{x}^{j}\right)^{2}+\frac{1}{2}\left(S_{z}^{j}\right)^{2}-1, \\
& \mathcal{S}_{21}^{j}=\frac{1}{2 i}\left\{S_{x}^{j}, S_{y}^{j}\right\}+\left(S_{x}^{j}\right)^{2}+\frac{1}{2}\left(S_{z}^{j}\right)^{2}-1,
\end{aligned}
$$

with $S_{ \pm}^{j}=S_{x}^{j} \pm i S_{y}^{j}$. From the spin-rotational invariance of the state $|\Phi\rangle_{\mathrm{VBS}}$ we get $\left\langle\left(S_{x}^{j}\right)^{2}\right\rangle_{\mathrm{VBS}}=$ $\left\langle\left(S_{y}^{j}\right)^{2}\right\rangle_{\mathrm{VBS}}=\left\langle\left(S_{z}^{j}\right)^{2}\right\rangle_{\mathrm{VBS}}=\frac{S(S+1)}{3}$ and, since $S=1$, we obtain $\left\langle\mathcal{S}_{00}^{j}\right\rangle_{\mathrm{VBS}}=\left\langle\mathcal{S}_{11}^{j}\right\rangle_{\mathrm{VBS}}=0$. Moreover, the spin-rotational invariance also implies that $\left\langle S_{\alpha}^{j} S_{\alpha^{\prime}}^{j}\right\rangle_{\mathrm{VBS}}$ remains the same constant $\forall \alpha \neq \alpha^{\prime}$. Then, for example, applying a global $\pi$-rotation about the $y$-axis to $|\Phi\rangle_{\text {VBS }}$ (i.e., the operation that maps $S_{z}^{j} \mapsto-S_{z}^{j}$ and $S_{y}^{j} \mapsto S_{y}^{j}$ ) we obtain $\left\langle S_{y}^{j} S_{z}^{j}\right\rangle_{\mathrm{VBS}}=-\left\langle S_{y}^{j} S_{z}^{j}\right\rangle_{\mathrm{VBS}}=0$, hence, $\left\langle\mathcal{S}_{\mu \nu}^{j}\right\rangle_{\mathrm{VBS}}=0$. Therefore, the state $|\Phi\rangle_{\mathrm{VBS}}(S=1, M=1)$ is maximally entangled when using the standard notion of entanglement $\left(P_{\mathfrak{h}}=0\right.$, for the algebra of all local observables $\left.\mathfrak{h}=\bigoplus_{j} \mathfrak{s u}(3)_{j}\right)$.

\section{APPENDIX C: RELATION BETWEEN PURITY IN THE LOCAL ALGEBRA AND THE} MEYER-WALLACH MEASURE OF ENTANGLEMENT

In Ref. [39], Meyer and Wallach define a measure of entanglement $Q$ for pure states of qubit systems, that is invariant under local unitary operations (local rotations). For this purpose, they first define the mapping $l_{j}(b)$ acting on product states as

$$
l_{j}(b)\left|b_{1}, \cdots, b_{N}\right\rangle=\delta_{b b_{j}}\left|b_{1}, \cdots, \hat{b}_{j}, \cdots, b_{N}\right\rangle,
$$

where $b$ and $b_{j}$ are either the states $\left|\frac{1}{2}\right\rangle$ or $\left|-\frac{1}{2}\right\rangle$, and $\hat{b}_{j}$ denotes the absence of the $j$-th qubit. On the other hand, any $N$-qubits pure quantum state can be written in the natural basis (z-component of the spin equal to $\pm \frac{1}{2}$ ) as

$$
|\psi\rangle=\sum_{i=1}^{2^{N-1}}\left[g_{i}^{j}\left|\frac{1}{2}\right\rangle_{j}+h_{i}^{j}\left|-\frac{1}{2}\right\rangle_{j}\right]\left|\phi_{i}\right\rangle,
$$


where $g_{i}^{j}$ and $h_{i}^{j}$ are complex coefficients, and the orthonormal states $\left|\phi_{i}\right\rangle$ of $N-1$ qubits (absence of the $j$-th qubit) are also written in the natural basis. Therefore, the action of $l_{j}(b)$ on $|\psi\rangle$ is

$$
\begin{aligned}
& l_{j}\left(\frac{1}{2}\right)|\psi\rangle=\sum_{i=1}^{2^{N-1}} g_{i}^{j}\left|\phi_{i}\right\rangle \\
& l_{j}\left(-\frac{1}{2}\right)|\psi\rangle=\sum_{i=1}^{2^{N-1}} h_{i}^{j}\left|\phi_{i}\right\rangle .
\end{aligned}
$$

Then, they define the entanglement $Q(|\psi\rangle)$ as

$$
Q(|\psi\rangle)=\frac{4}{N} \sum_{j=1}^{N} D\left(l_{j}\left(\frac{1}{2}\right)|\psi\rangle, l_{j}\left(-\frac{1}{2}\right)|\psi\rangle\right)
$$

where the distance between two quantum states $|u\rangle=\sum u_{i}\left|\phi_{i}\right\rangle$ and $|v\rangle=\sum v_{i}\left|\phi_{i}\right\rangle$ is

$$
D(u, v)=\frac{1}{2} \sum_{i, j}\left|u_{i} v_{j}-u_{j} v_{i}\right|^{2}
$$

Therefore,

$$
D\left(l_{j}\left(\frac{1}{2}\right)|\psi\rangle, l_{j}\left(-\frac{1}{2}\right)|\psi\rangle\right)=\frac{1}{2} \sum_{i, i^{\prime}}\left|g_{i}^{j} h_{i^{\prime}}^{j}-g_{i^{\prime}}^{j} h_{i}^{j}\right|^{2}=\sum_{i, i^{\prime}}\left[\left|g_{i}^{j}\right|^{2}\left|h_{i^{\prime}}^{j}\right|^{2}-\left(g_{i}^{j} h_{i^{\prime}}^{j}\right)\left(h_{i}^{j} g_{i^{\prime}}^{j}\right)^{*}\right],
$$

where * denotes complex conjugate. After some simple calculations we obtain the following relations

$$
\begin{gathered}
\sum_{i=1}^{2^{N-1}}\left|g_{i}^{j}\right|^{2}=\left\langle\psi\left|\left(\frac{1+\sigma_{z}^{j}}{2}\right)\right| \psi\right\rangle, \\
\sum_{i=1}^{2^{N-1}}\left|h_{i}^{j}\right|^{2}=\left\langle\psi\left|\left(\frac{1-\sigma_{z}^{j}}{2}\right)\right| \psi\right\rangle, \\
\sum_{i=1}^{2^{N-1}} g_{i}^{j}\left(h_{i}^{j}\right)^{*}=\left\langle\psi\left|\sigma_{-}^{j}\right| \psi\right\rangle,
\end{gathered}
$$

and the distance becomes $D\left(l_{j}\left(\frac{1}{2}\right)|\psi\rangle, l_{j}\left(-\frac{1}{2}\right)|\psi\rangle\right)=\frac{1}{4}\left[1-\left\langle\sigma_{z}^{j}\right\rangle^{2}-\left\langle\sigma_{x}^{j}\right\rangle^{2}-\left\langle\sigma_{y}^{j}\right\rangle^{2}\right]$. Since $Q(|\psi\rangle)$ contains a sum over all qubits (see Eq. (C3)), we finally obtain

$$
Q(|\psi\rangle)=1-\frac{1}{N} \sum_{j=1}^{N}\left[\left\langle\sigma_{z}^{j}\right\rangle^{2}+\left\langle\sigma_{x}^{j}\right\rangle^{2}+\left\langle\sigma_{y}^{j}\right\rangle^{2}\right]=1-P_{\mathfrak{h}}(|\psi\rangle)
$$

where $P_{\mathfrak{h}}$ is the purity relative to the local algebra $\mathfrak{h}_{l o c}=\bigoplus_{j=1}^{N} \mathfrak{s u}(2)_{j}$ defined in Section 


\section{APPENDIX D: CLASSICAL LIMIT IN THE LMG MODEL}

As we mentioned in Section $\nabla$, some critical properties of the LMG, such as the order parameter or the ground state energy per particle in the thermodynamic limit, may be obtained using a semiclassical approach. In this section we sketch a rough analysis of why such approximation is valid (for a more extensive analysis, see Ref. [45]).

We first define the collective operators

$$
E_{\left(\sigma, \sigma^{\prime}\right)}=\sum_{k=1}^{N} c_{k \sigma}^{\dagger} c_{k \sigma^{\prime}}
$$

where $\sigma, \sigma^{\prime}=\uparrow$ or $\downarrow$ and the fermionic operators $c_{k \sigma}^{\dagger}\left(c_{k \sigma}\right)$ have been defined in Section $\nabla$ The collective operators satisfy the $\mathfrak{u}(2)$ commutation relations (Section $\amalg \mathrm{C}$ ); that is

$$
\left[E_{\left(\sigma, \sigma^{\prime}\right)}, E_{\left(\sigma^{\prime \prime}, \sigma^{\prime \prime \prime}\right)}\right]=\delta_{\sigma^{\prime} \sigma^{\prime \prime}} E_{\left(\sigma, \sigma^{\prime \prime \prime}\right)}-\delta_{\sigma \sigma^{\prime \prime \prime}} E_{\left(\sigma^{\prime \prime}, \sigma^{\prime}\right)}
$$

If the number of degenerate levels $N$ is very large, it is useful to define the intensive collective operators $\hat{E}_{\left(\sigma, \sigma^{\prime}\right)}=E_{\left(\sigma, \sigma^{\prime}\right)} / N$, with commutation relations

$$
\left[\hat{E}_{\left(\sigma, \sigma^{\prime}\right)}, \hat{E}_{\left(\sigma^{\prime \prime}, \sigma^{\prime \prime \prime}\right)}\right]=\frac{1}{N}\left(\delta_{\sigma^{\prime} \sigma^{\prime \prime}} \hat{E}_{\left(\sigma, \sigma^{\prime \prime \prime}\right)}-\delta_{\sigma \sigma^{\prime \prime \prime}} \hat{E}_{\left(\sigma^{\prime \prime}, \sigma^{\prime}\right)}\right)
$$

Therefore, the intensive collective operators commute in the limit $N \rightarrow \infty$, they are effectively classical and can be simultaneously diagonalized. Similarly, the intensive angular momentum

operators $J_{x} / N=\left(\hat{E}_{(\uparrow, \downarrow)}+\hat{E}_{(\downarrow, \uparrow)}\right) / 2, J_{y} / N=\left(\hat{E}_{(\uparrow, \downarrow)}-\hat{E}_{(\downarrow, \uparrow)}\right) / 2 i$, and $J_{z} / N=\left(\hat{E}_{(\uparrow, \uparrow)}-\hat{E}_{(\downarrow, \downarrow)}\right) / 2$ (with $J_{\alpha}$ defined in Eqs. (24), (25), and (26) commute with each other in the thermodynamic limit, so they can be thought of as the angular momentum operators of a classical system.

Since the intensive LMG Hamiltonian $H / N$, with $H$ given in Eq. 29), can be written in terms of the intensive angular momentum operators, it can be regarded as the Hamiltonian describing a classical system. The ground state of the LMG model $|g\rangle$ is then an eigenstate of such intensive operators when $N \rightarrow \infty:\left(J_{\alpha} / N\right)|g\rangle=j_{\alpha}|g\rangle, j_{\alpha}$ being the corresponding eigenvalue. In other words, when obtaining some expectation values of intensive operators such as $J_{\alpha} / N$ or $H / N$ the ground state $|g\rangle$ can be pictured as a classical angular momentum with fixed coordinates in the three-dimensional space (see Fig. 33).

This point of view makes it clear why such operators ought to be intensive. Otherwise, such a classical limit is not valid and terms of order 1 would be important for the calculations of the properties of the LMG model. Obviously, all these concepts can be extended to more complicated 
Hamiltonians such as the extended LMG model, or even Hamiltonians including interactions of higher orders as in [45].

[1] S. Sachdev, Quantum Phase Transitions (Cambridge University Press, Cambridge, U.K., 1999).

[2] E. Lieb, T. Schultz, and D. Mattis, Ann. Phys. (N. Y.) 16, 406 (1961).

[3] P. Pfeuty, Ann. Phys. (N. Y.) 57, 79 (1970).

[4] E. Barouch and B. McCoy, Phys. Rev. A 3, 786 (1971).

[5] M. Greiner, O. Mandel, T. Esslinger, T. W. Hänsch, and I. Bloch, Nature 415, 39 (2002).

[6] E. Schrödinger, Naturwissenschaften 23, 807 (1935). English translation available in Proc. Am. Phil. Soc. 124, 323 (1980).

[7] T. J. Osborne and M. A. Nielsen, Quant. Inf. Process. 1, 45 (2002); Phys. Rev. A 66, 032110 (2002).

[8] A. Osterloh, L. Amico, G. Falci, and R. Fazio, Nature 416, 608 (2002).

[9] M. Arnesen, S. Bose, and V. Vedral, Phys. Rev. Lett. 87, 290 (2001).

[10] G. Vidal, J. I. Latorre, E. Rico, and A. Kitaev, Phys. Rev. Lett. 90, 227902 (2003).

[11] H. Barnum, E. Knill, G. Ortiz, R. Somma, and L. Viola, quant-ph/0305023, Phys. Rev. Lett. 92, 107902 (2004).

[12] F. Verstraete, M. A. Martin-Delgado, and J. I. Cirac, quant-ph/0311087

[13] C. H. Bennett, D. P. DiVincenzo, J. Smolin, and W. Wootters, Phys. Rev. A 54, 3824 (1996).

[14] W. Wootters, Phys. Rev. Lett. 80, 2245 (1998).

[15] H. Barnum, E. Knill, G. Ortiz, and L. Viola, Phys. Rev. A 68, 032308 (2003).

[16] C. D. Batista and G. Ortiz, Phys. Rev. Lett. 86, 1082 (2001).

[17] C. D. Batista and G. Ortiz, Adv. Phys. 53, 1 (2004).

[18] The definition of entanglement-like notions in systems composed of indistinguishable particles has attracted considerable attention recently. See for instance K. Eckert, J. Schliemann, D. Bruss, and M. Lewenstein, Ann. Phys. (N. Y.) 299, 88 (2002).

[19] The reduced state is the linear functional on the space spanned by the distinguished observables whose values on those observables are the expectation values given by the quantum state in question.

[20] Recall that an extremal point in a convex set is one that cannot be written as a non-trivial convex combination $\sum_{i} p_{i} x_{i}, p_{i} \geq 0, \sum_{i} p_{i}=1$, of other points $x_{i}$ in the set.

[21] H. Lipkin, N. Meshkov, and A. Glick, Nucl. Phys. 62, 188 (1965). 
[22] G. Ortiz, R. Somma, J. Dukelsky, and R. Rombouts, cond-mat/0407429

[23] E. Knill, R. Laflamme, and L. Viola, Phys. Rev. Lett. 84, 2525 (2000).

[24] S. De Filippo, Phys. Rev. A 62, 052307 (2000).

[25] L. Viola, E. Knill, and R. Laflamme, J. Phys. A 34, 7067 (2001).

[26] P. Zanardi, Phys. Rev. Lett. 87, 077901 (2001).

[27] P. Zanardi, D. A. Lidar, and S. Lloyd, Phys. Rev. Lett. 92, 060402 (2004).

[28] H. Georgi, Lie Algebras in Particle Physics (Perseus Books, Reading, Massachusetts, 1999).

[29] B. C. Hall, Lie Groups, Lie Algebras, and Representations (Springer-Verlag, New York, 2003).

[30] J. E. Humphreys, Introduction to Lie Algebras and Representation Theory (Springer-Verlag, New York, 1972).

[31] W. Fulton and J. Harris, Representation theory: A first course (Springer-Verlag, New York, 1991).

[32] R. Gilmore, Rev. Mex. de Fis. 23, 143 (1974).

[33] A. Perelomov, Generalized Coherent States and their Applications (Springer-Verlag, Berlin, 1985).

[34] W. M. Zhang, D. H. Feng, and R. Gilmore, Rev. Mod. Phys. 62, 867 (1990).

[35] R. Delbourgo, J. Phys. A 10, 1837 (1977).

[36] R. Delbourgo and J. R. Fox, J. Phys. A, 10 L233 (1977).

[37] A. Einstein, B. Podolsky, and N. Rosen, Phys. Rev. 47, 777 (1935).

[38] H. J. Briegel and R. Raussendorf, Phys. Rev. Lett. 86, 910 (2001).

[39] D. A. Meyer and N. R. Wallach, J. Math. Phys. 43, 4273 (2002).

[40] G. K. Brennen, Quantum Inf. Comp. 3, 619 (2003).

[41] L. Viola, H. Barnum, E. Knill, R. Somma, G. Ortiz, quant-ph/0403044

[42] P. Zanardi, Phys. Rev. A 65, 042101 (2002).

[43] R. Somma, G. Ortiz, J. E. Gubernatis, E. Knill, and R. Laflamme, Phys. Rev. A 65, 042323 (2002).

[44] R. Somma, G. Ortiz, H. Barnum, E. Knill, and L. Viola, in preparation.

[45] R. Gilmore, Catastrophe Theory for Scientists and Engineers (Wiley-Interscience, New York, 1981).

[46] Similarly, there are ways to rewrite Eq. 23] in terms of some algebras that are products of $N$ "local" $\mathfrak{s u}(2)$ 's. This is related to the approach taken in J. Vidal, G. Palacios, and R. Mosseri, Phys. Rev. A 69, 022107 (2004) which uses a standard entanglement measure, the concurrence, to study phase transitions in the LMG model. Such an approach implies algebras which are much larger than the $\mathfrak{s u}(2)$ subalgebra generated by the LMG Hamiltonians, and we would argue that the additional detail embodied in the partitions into "local" spins is distracting rather than helpful if one wants to study 
phase transitions in this particular model. Indeed, the critical behavior of the LMG model is governed by the expectations of collective operators.

[47] R. Gilmore and D. H. Feng, Phys. Lett. B 76, 26 (1978).

[48] P. Jordan and E. Wigner, Z. Phys. 47, 631 (1928).

[49] M. Takahashi, Thermodynamics of one-dimensional solvable models (Cambridge University Press, Cambridge, 1999).

[50] F. Verstraete and J. I. Cirac, quant-ph/0311130

[51] U. Glaser, H. Buttner, and H. Fehske, Phys. Rev. A 68, 032318 (2003).

[52] A. Yu.Kitaev, Ann. Phys. 303, 2 (2003); M. H. Freedman, A. Yu. Kitaev, M. J. Larsen, Z. Wang, Bull. Amer. Math. Soc., 40, 31 (2002). 